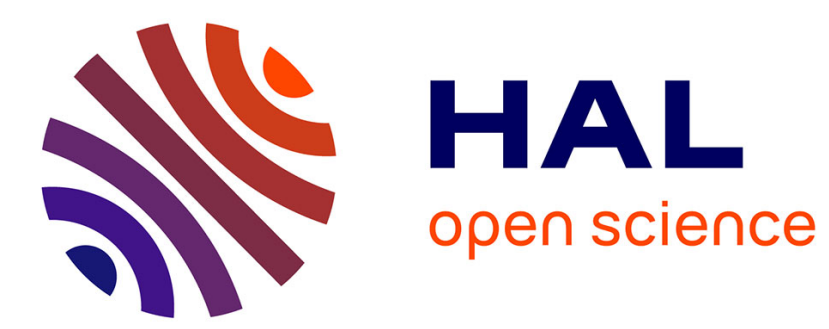

\title{
On the Computation of Set-Induced Control Lyapunov Functions for Continuous-Time Systems
}

Mirko Fiacchini, Christophe Prieur, Sophie Tarbouriech

\section{To cite this version:}

Mirko Fiacchini, Christophe Prieur, Sophie Tarbouriech. On the Computation of Set-Induced Control Lyapunov Functions for Continuous-Time Systems. SIAM Journal on Control and Optimization, 2015, 53 (3), pp.1305-1327. 10.1137/130934908 . hal-01133446

\section{HAL Id: hal-01133446 https://hal.science/hal-01133446}

Submitted on 2 Jun 2015

HAL is a multi-disciplinary open access archive for the deposit and dissemination of scientific research documents, whether they are published or not. The documents may come from teaching and research institutions in France or abroad, or from public or private research centers.
L'archive ouverte pluridisciplinaire HAL, est destinée au dépôt et à la diffusion de documents scientifiques de niveau recherche, publiés ou non, émanant des établissements d'enseignement et de recherche français ou étrangers, des laboratoires publics ou privés. 


\title{
ON THE COMPUTATION OF SET-INDUCED CONTROL LYAPUNOV FUNCTIONS FOR CONTINUOUS-TIME SYSTEMS
}

\author{
MIRKO FIACCHINI*, CHRISTOPHE PRIEUR* ${ }^{*}$, AND SOPHIE TARBOURIECH ${ }^{\dagger}$
}

\begin{abstract}
The paper presents a computation-oriented method for characterizing and obtaining local control Lyapunov functions induced by particular star-shaped nonconvex sets for continuous-time nonlinear systems with bounded inputs. For a given set, the necessary and sufficient conditions for the induced function to be a nonconvex local control Lyapunov function are provided. The related convex problems for computing the exact region in which the function is decreasing and the optimal control input are presented. The results are applied to the Brockett integrator.
\end{abstract}

Key words. Invariant sets, viability theory, convex analysis, nonlinear systems.

1. Introduction. Although the first, pioneering contributions on invariance and settheory in control appear in the seventies, see [5], these techniques gain and increase their popularity since the nineties, after the publication of $[7,8]$. The importance of invariant and contractive sets resides on their connection with stability and convergence of dynamical systems, see the recent monograph for an overview [9] on the topic. It is proved in [7], for instance, that in the context of linear parametric uncertain systems, polyhedral Lyapunov functions, i.e. positive definite homogeneous functions induced by polytopes, represent a universal class of Lyapunov functions. In the context of continuous-time nonlinear systems, the characterization of the convex polyhedral Lyapunov functions has been considered in [29]. More recently, the analogous results for linear discrete-time switched systems has been proved to hold for a particular class of nonconvex sets-induced functions, see [20]. The fact that stabilizability does not imply the existence of a convex Lyapunov function had been proved for both continuous and discrete-time switched linear systems in [10]. A peculiarity that makes particularly interesting set-theoretic methods is their relation with computationoriented techniques, as convex analysis and optimization, that permit their practical application. In the last two decades, many research efforts have been directed to analyze and apply set-theoretic and invariance methods in control, in the linear context, see [23, 28], as well as in the nonlinear one, see [17, 14, 1, 18, 19] for discrete-time systems and [13, 27, 25, 39, 22, 24] for particular classes of continuous-time and hybrid nonlinear systems.

The objective of this paper is to exploit the properties of viability theory to propose a computation-oriented method to characterize and obtain nonconvex set-induced control Lyapunov functions for a class of nonlinear continuous-time systems. Viability theory provides a complete theoretical characterization of invariance and set-induced Lyapunov functions in the differential inclusion framework. Viability is tightly related to the work of Aubin and co-authors, see $[4,3,2]$. The results provided by viability theory together with the methods and techniques proper of convex analysis and optimization, see [6,11,35, 37], are the basis of the approach.

In this paper, whose preliminary version is [21], we propose a computation-oriented method to characterize and compute nonconvex set-induced control Lyapunov functions for continuous-time nonlinear systems affine in the input with bounded input. Nonconvex homogeneous Lyapunov functions, in particular piecewise quadratic ones, have been employed to prove stability for continuous-time affine systems, see [34]. The nonconvex sets considered

\footnotetext{
${ }^{*}$ GIPSA-lab, Grenoble Campus, 11 rue des Mathématiques, BP 46, 38402 Saint Martin d'Hères Cedex, France. \{mirko.fiacchini, christophe.prieur\}@gipsa-lab.fr

${ }^{\dagger}$ CNRS, LAAS, 7 avenue du Colonel Roche, F-31400 Toulouse, France and Univ. de Toulouse, LAAS, F-31400 Toulouse, France. tarbour@laas. fr
} 
here are a subclass of the star-shaped sets that have been widely studied by Rubinov and coauthors, see for instance [36], and employed in [33, 31, 20]. We consider in particular the computationally suitable star-shaped sets determined by the intersection and union operators and a finite number of half-spaces containining the origin in their interior. Given one of these nonconvex sets, and the induced polyhedral function as control Lyapunov candidate for the nonlinear system, a necessary and sufficient condition for the function to be decreasing at a point along a trajectory of the system is provided. Such a control Lyapunov function yields local exponential stabilizability for the nonlinear constrained system. The exact region of the state space in which the decreasing of the polyhedral Lyapunov function, and hence exponential stability, is ensured by admissible inputs, is characterized. The optimal control input guaranteeing maximal decreasing is obtained. Particular attention is devoted to the computational issues of such functions and control laws. Finally, the proposed method is applied to the problem of characterizing the region of local exponential stabilizability for the Brockett integrator, to show that nonconvexity might be necessary to have exponential stabilizability of nonlinear systems.

The paper is organized as follows: Section II presents the problem statement, Section III recalls some definitions and results on viability theory. Section IV presents the nonconvex sets employed and the induced homogeneous functions. In Section V the main results on polyhedral control Lyapunov functions are stated. Section VI is devoted to the computational issues. In Section VII the method is applied to the Brockett integrator. The paper ends with a section of conclusions.

Notation. Given $n \in \mathbb{N}$, define $\mathbb{N}_{n}=\{x \in \mathbb{N}: 1 \leq x \leq n\}$. Given $A \in \mathbb{R}^{n \times m}$, $A_{i}$ with $i \in \mathbb{N}_{n}$ denotes its $i$-th row. Given a set $D$ and a scalar $\alpha \geq 0$, denote the scalar multiple of $D$ as $\alpha D=\{\alpha x: x \in D\}$. The interior of $D$ is denoted as $\operatorname{int}(D)$, with $\mathscr{S}(D)$ the set of subsets of $D$. The unit ball in $\mathbb{R}^{n}$ is $\mathbf{B}^{n}=\left\{x \in \mathbb{R}^{n}:\|x\|_{2} \leq 1\right\}$. Given a set-valued map $F: \mathbb{R}^{n} \rightarrow \mathscr{S}\left(\mathbb{R}^{m}\right)$, its domain is $\operatorname{dom}(F)=\left\{x \in \mathbb{R}^{n}: F(x) \neq \emptyset\right\}$.

2. Problem statement. Consider the continuous-time system given by

$$
\dot{x}(t)=f(x(t), u(t)), \quad \text { for almost all } t \geq 0,
$$

where $x(t) \in \mathbb{R}^{n}$ is the state at time $t$, and with input bounds $u(t) \in U(x(t))$. This class of systems can be represented by using the modeling framework referred to as differential inclusion, characterized by the system

$$
\dot{x}(t) \in F(x(t)), \quad \text { for almost all } t \geq 0,
$$

with set-valued map $F: \mathbb{R}^{n} \rightarrow \mathscr{S}\left(\mathbb{R}^{n}\right)$. Indeed, the constrained control systems (2.1) can be expressed as a differental inclusion with

$$
F(x)=f(x, U(x))=\left\{y \in \mathbb{R}^{n}: y=f(x, u), u \in U(x)\right\}
$$

see [3]. Differential inclusions can be also used to approximate nonlinear systems $\dot{x}(t)=$ $f(x(t))$, provided that $f(x) \in F(x)$ for all $x \in \mathbb{R}^{n}$, see [4, 3]. This characterization of the system (2.1) is taken into account in this paper to apply results from viability theory, see [3]. The modeling framework considered in this paper is summarized in the following assumption.

ASSUMPTION 2.1. Assume that the system dynamics is given by (2.1) where $f(x, u)=$ $g(x)+h(x) u$ with $u \in U(x)$ where

$$
U(x)=\left\{u \in \mathbb{R}^{m}: M_{j} u \leq N_{j}(x), \forall j \in \mathbb{N}_{n_{u}}\right\}
$$


and $g, N_{j}$, with $j \in \mathbb{N}_{n_{u}}$, are continuous with linear growth and $h$ is continuous and bounded.

REMARK 2.2. The satisfaction of Assumption 2.1 is sufficient for the system to be an affine control system and then also a Marchaud system, see [3]. A set-valued map is Marchaud if its graph and domain are closed, the values $F(x)$ are convex and the growth of $F$ is linear. Although many results based on viability theory apply to more general systems, the case considered is relatively general and rather suitable for computational purposes.

The objective is to design a computation-oriented method for obtaining set-induced local control Lyapunov functions, estimations of the basin of attraction and control inputs such that the nonlinear continuous-time system (2.1) with state-dependent polytopic bounds on the input is exponentially stable. As already pointed out, this is equivalent to consider the system (2.2) with set-valued map defined in (2.3). Moreover, the explicit formulation of the optimal control law, maximizing the decreasing of the induced Lyapunov function, is provided.

REMARK 2.3. Assumption 2.1 is equivalent to consider a system $\dot{x}(t)=f(x(t), u(t))$ with bounds in the input such that $U(x)$ is polytopic and $U$ is a Marchaud map, see [3], with $F$ as in (2.3).

3. Viability theory and Lyapunov functions. We recall some definitions and results on viability theory, which is strongly associated to the research of Aubin and co-authors, see $[4,3,2]$. Many of those results are developed in the cited works, and references therein, under assumptions which are more general than those required in this paper.

DEFINITION 3.1. Let $V: \mathbb{R}^{n} \rightarrow \mathbb{R} \cup\{ \pm \infty\}$ be a nontrivial extended function and $x$ belong to its domain. For all $y \in \mathbb{R}^{n}$, define the extended function

$$
D_{\uparrow} V(x)(y)=\liminf _{h \rightarrow 0^{+}, v \rightarrow y} \frac{V(x+h v)-V(x)}{h},
$$

and refer to function $D_{\uparrow} V(x)$ as the contingent epiderivative of $V$ at $x$ and say that the function $V$ is contingently epidifferentiable at $x$ if for any $y \in \mathbb{R}^{n}, D_{\uparrow} V(x)(y)>-\infty$ (or, equivalently, if $\left.D_{\uparrow} V(x)(0)=0\right)$.

Geometrically, $D_{\uparrow} V(x)(y)$ is the extended function whose epigraph is the contingent cone (and also the tangent one if $V$ is convex and closed) of the epigraph of $V$ at $(x, V(x))$, see [3].

REMARK 3.2. The contingent epiderivative is equal to the (lower) Dini derivative if $V$ is Lipschitz around a point $x$ of its domain (Proposition 9.1.5 in [3]) and it is the support function of the generalized gradient (and also of the subdifferential if $V$ is convex), see [15], under certain regularity assumptions. We use the contingent epiderivative to maintain the coherence with viability theory and to deal also with nonconvex homogeneous functions.

In this paper, the properties and theorems concerning the Lyapunov functions for differential inclusions (2.2), provided in [3, 2], are applied. We recall the characterization of Lyapunov functions in the context of viability theory. Given a differential equation

$$
\dot{w}(t)=-\varphi(w(t)),
$$

where $\varphi: \mathbb{R}_{+} \rightarrow \mathbb{R}$ is continuous with linear growth and a nonnegative extended function $V: \mathbb{R}^{n} \rightarrow \mathbb{R}_{+} \cup\{+\infty\}$, the objective is to provide a condition for the existence of a solution $x$ to the differential inclusion (2.2) ensuring

$$
\forall t \geq 0, \quad V(x(t)) \leq w(t), \quad w(0)=V(x(0)),
$$

with $w$ solution to (3.2). Clearly, if $\varphi$ is selected such that $w$ converges to zero, then also $V$ converges to zero and $x$ converges to $x \in \operatorname{dom}(V)$ such that $V(x)=0$. The general definition of Lyapunov function in the context of differential inclusion follows. 
DEFINITION 3.3. A nonnegative contingently epidifferentiable extended function $V$ is a Lyapunov function of $F$ associated with a function $\varphi: \mathbb{R}_{+} \rightarrow \mathbb{R}$ if and only if $V$ is a solution to the contingent Hamilton-Jacobi inequalities

$$
\inf _{y \in F(x)} D_{\uparrow} V(x)(y)+\varphi(V(x)) \leq 0, \quad \forall x \in \operatorname{dom}(V) .
$$

We recall that contingently epidifferentiable means that for all $x \in \operatorname{dom}(V)$, for all $y \in \mathbb{R}^{n}$, $D_{\uparrow} V(x)(y)>-\infty$ and that $D_{\uparrow} V(x)(y)<+\infty$ for at least a $y \in \mathbb{R}^{n}$.

THEOREM 3.4. Consider the nonnegative contingently epidifferentiable lower semicontinuous extended function $V$ and the Marchaud map $F: \mathbb{R}^{n} \rightarrow \mathscr{S}\left(\mathbb{R}^{n}\right)$. Then $V$ is a Lyapunov function of $F$ associated with $\varphi$ if and only if for any $x_{0} \in \operatorname{dom}(V)$, there exist solutions $x$ to (2.2) and $w$ to (3.2) satisfying property (3.3).

Theorem 3.4, proved in [3], is instrumental to compute control Lyapunov functions as done in this paper.

4. Nonconvex polyhedral sets and functions. Consider first a polytope in the state space containing the origin in its interior, $\Omega=\left\{x \in \mathbb{R}^{n}: H x \leq 1\right\}$, with $H \in \mathbb{R}^{n_{h} \times n}$, and the system (2.1) such that Assumption 2.1 holds. The gauge function is introduced here, see $[35,37]$ for instance.

DEFINITION 4.1. Given a compact, convex set $\Omega \in \mathbb{R}^{n}$ with $0 \in \operatorname{int}(\Omega)$, the gauge function of $\Omega$ at $x \subseteq \mathbb{R}^{n}$ is defined by $\Psi_{\Omega}(x)=\min _{\alpha>0}\{\alpha \in \mathbb{R}: x \in \alpha \Omega\}$.

For polytopic $\Omega$ containing the origin in its interior, the gauge function is

$$
\Psi_{\Omega}(x)=\min _{\alpha \geq 0}\left\{\alpha \in \mathbb{R}: H_{j} x \leq \alpha, \forall j \in \mathbb{N}_{h_{h}}\right\}=\max _{j \in \mathbb{N}_{n_{h}}}\left\{H_{j} x\right\} .
$$

Consider the set $\bar{\Theta} \subseteq \mathbb{R}^{n}$ defined as

$$
\bar{\Theta}=\left\{x \in \mathbb{R}^{n}: \exists j \in \mathbb{N}_{n_{\bar{h}}} \text {, s.t. } \bar{H}_{j} x \leq 1\right\},
$$

see an example in Figure 1 The closed, possibly nonconvex, set $\bar{\Theta}$ contains the origin in its interior and is given by the union of the half-spaces defined by the inequalities $\bar{H}_{j} x \leq 1$ with $j \in \mathbb{N}_{h_{\tilde{h}}}$.

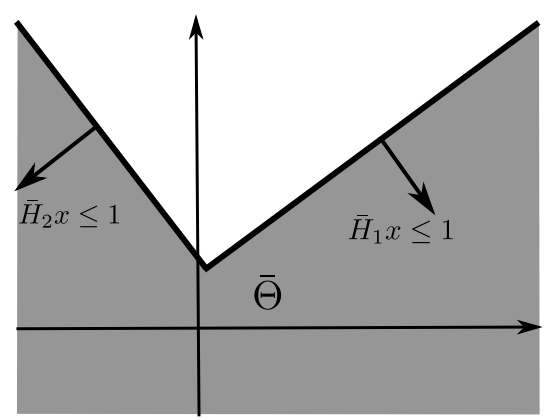

FIG. 1. Nonconvex set $\bar{\Theta}$ with $n_{h}=2$.

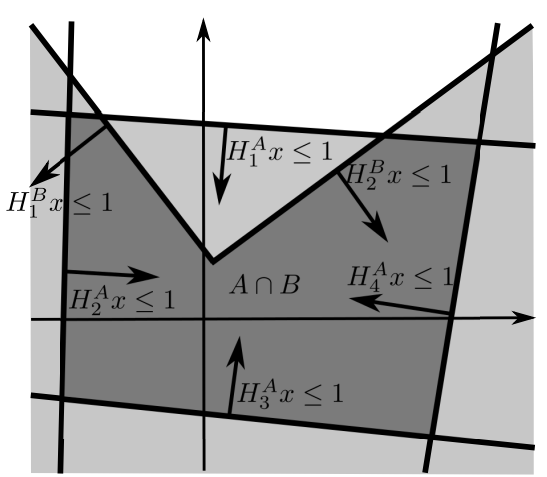

FIG. 2. Nonconvex set $A \cap B$ as in Example 4.4.

Given $\bar{\Theta}$, we define over $\mathbb{R}^{n}$ the following homogeneous function

$$
\Phi_{\bar{\Theta}}(x)=\min _{\alpha}\left\{\alpha \in \mathbb{R}: \exists j \in \mathbb{N}_{n_{\bar{h}}} \text {, s.t. } \bar{H}_{j} x \leq \alpha\right\},
$$


which is the analogous of the gauge function for a nonconvex set $\bar{\Theta}$ with $0 \in \operatorname{int}(\bar{\Theta})$. Notice that $\Phi_{\bar{\Theta}}$ is greater than 1 if and only if $x \notin \bar{\Theta}$ and the following equality holds

$$
\Phi_{\bar{\Theta}}(x)=\min _{i \in \mathbb{N}_{n_{\bar{h}}}}\left\{\bar{H}_{i} x\right\} .
$$

The analogy with the gauge function as in Definition 4.1 and equation (4.1) is evident. For polytopic sets $\Omega$ with $0 \in \operatorname{int}(\Omega)$ we pose $\Phi_{\Omega}(x)=\Psi_{\Omega}(x)$ for all $x \in \mathbb{R}^{n}$. Then the function $\Phi_{\Theta}$ can be seen as an extension of the gauge function related to particular sets $\Theta$, possibly nonconvex. In the following, the formal definition and properties of $\Phi_{\Theta}$ are given.

DEFINITION 4.2. Denote with $\mathscr{C}\left(\mathbb{R}^{n}\right)$ the subsets of $\mathbb{R}^{n}$ that can be expressed by using the intersection and the union operators and a finite number of closed half-spaces in $\mathbb{R}^{n}$ containing the origin in their interior.

Clearly any closed half-space containing the origin in its interior belongs to $\mathscr{C}\left(\mathbb{R}^{n}\right)$. Moreover, for any $B, C \in \mathscr{C}\left(\mathbb{R}^{n}\right)$, we have that $B \cup C \in \mathscr{C}\left(\mathbb{R}^{n}\right)$ and $B \cap C \in \mathscr{C}\left(\mathbb{R}^{n}\right)$. The following definition permits to construct functions $\Phi_{\Theta}$ related to any set $\Theta \in \mathscr{C}\left(\mathbb{R}^{n}\right)$.

Definition 4.3. Given the half-space $A=\left\{x \in \mathbb{R}^{n}: H x \leq 1\right\}$, with $H \in \mathbb{R}^{1 \times n}$, define $\Phi_{A}(x)=H x$. Given two sets $B, C \in \mathscr{C}\left(\mathbb{R}^{n}\right)$ define $\Phi_{B \cup C}(x)=\min \left\{\Phi_{B}(x), \Phi_{C}(x)\right\}$ and $\Phi_{B \cap C}(x)=\max \left\{\Phi_{B}(x), \Phi_{C}(x)\right\}$.

Then, the function $\Phi_{\Theta}$ can be defined for every set $\Theta \in \mathscr{C}\left(\mathbb{R}^{n}\right)$ and its domain is $\mathbb{R}^{n}$, being it finite at every $x \in \mathbb{R}^{n}$.

EXAMPLE 4.4. Given a polytope $A=\left\{x \in \mathbb{R}^{n}: H_{i}^{A} x \leq 1, \forall i \in \mathbb{N}_{a}\right\}$ and a set $B=\{x \in$ $\mathbb{R}^{n}: \exists j \in \mathbb{N}_{b}$, s.t. $\left.H_{j}^{B} x \leq 1\right\}$, we have that $\Phi_{A \cap B}(x)=\max \left\{\max _{i \in \mathbb{N}_{a}}\left\{H_{i}^{A} x\right\}, \min _{j \in \mathbb{N}_{b}}\left\{H_{j}^{B} x\right\}\right\}$, and $\Phi_{A \cup B}(x)=\min \left\{\max _{i \in \mathbb{N}_{a}}\left\{H_{i}^{A} x\right\}, \min _{j \in \mathbb{N}_{b}}\left\{H_{j}^{B} x\right\}\right\}$. The set $A \cap B$ is represented in Figure 2,

REMARK 4.5. The sets in $\mathscr{C}\left(\mathbb{R}^{n}\right)$ form a particular subfamily of the called star-shaped sets, introduced and characterized in the work by Rubinov and co-authors, see for instance [36]. Also the function $\Phi_{\Theta}$ is analogous to the gauge function of a star-shape set as defined and employed by Rubinov. Notice, nevertheless, that our definition is slightly different from the gauge one, since it may take negative values. Despite the substantial analogy, we avoid imposing its nonnegativity for simplicity as well as for maintaining the consistency in its relation with the contingent epiderivative from viability theory.

The intuitive definition provided above for the set $\bar{\Theta}$ and for the polytopes $\Omega$ are recovered by using Definition 4.3. In fact, for all $\Theta \in \mathscr{C}\left(\mathbb{R}^{n}\right), \Phi_{\Theta}$ is given by nested minima and maxima of linear functions of the state.

We provide below some properties of the function $\Phi_{\Theta}(x)$ for seek of completeness. We refer the reader to the work by Rubinov and co-authors for analogous considerations concerning the gauge functions of star-shaped sets, see [36].

Proposition 4.6. Given $\Theta \in \mathscr{C}\left(\mathbb{R}^{n}\right)$, the function $\Phi_{\Theta}: \mathbb{R}^{n} \rightarrow \mathbb{R}$ is continuous, positively homogeneous of degree 1 and such that

$$
x \in \Theta \quad \Leftrightarrow \quad \Phi_{\Theta}(x) \leq 1 .
$$

Moreover, $\Phi_{\Theta}$ is positive definite if and only if $\Theta$ is bounded.

Proof. Homogeneity is due to the fact that functions min and max are positively homogeneous of degree 1, i.e. $\min _{i}\left\{H_{i} \alpha x\right\}=\alpha \min _{i}\left\{H_{i} x\right\}$ and $\max _{i}\left\{H_{i} \alpha x\right\}=\alpha \max _{i}\left\{H_{i} x\right\}$ for all $\alpha \geq 0$. From Definition 4.3, $\Phi_{\Theta}$ can be expressed as nested maxima and minima of linear functions of the state. Continuity follows from the fact that max and min preserve continuity and linear functions are evidently continuous. Equivalence (4.4) is proved by induction. First notice that, if $\Theta$ is given by one half-space, then the condition is satisfied by Definition 4.3. 
Assume now that (4.4) holds for two sets $B, C \in \mathscr{C}\left(\mathbb{R}^{n}\right)$, we prove that this implies $x \in B \cup C$ if and only if $\Phi_{B \cup C}(x) \leq 1$. Indeed, we have

$$
\begin{gathered}
x \in B \cup C \quad \Leftrightarrow \quad x \in B \text { or } x \in C \quad \Leftrightarrow \quad \Phi_{B}(x) \leq 1 \text { or } \Phi_{C}(x) \leq 1 \quad \Leftrightarrow \\
\Leftrightarrow \min \left\{\Phi_{B}(x), \Phi_{C}(x)\right\} \leq 1 \quad \Leftrightarrow \quad \Phi_{B \cup C}(x) \leq 1 .
\end{gathered}
$$

Analogous considerations yield to prove that $x \in B \cap C$ if and only if $\Phi_{B \cap C}(x) \leq 1$. Then using the rule described in Definition 4.3 for constructing the set $\Theta$, the equivalence (4.4) is proved. Finally we prove that $\Phi_{\Theta}$ is positive definite if and only if $\Theta$ is bounded. By construction $\Phi_{\Theta}(0)=0$. To prove sufficiency, assume that $\Phi_{\Theta}$ is positive definite and consider any $x \neq 0$. Then $\Phi_{\Theta}(x)>0$ and, from positive homogeneity, there exists $\alpha=\alpha(x)$ such that $\Phi_{\Theta}(\alpha x)>$ 1 , which means that $\alpha x \notin \Theta$. Thus, given $\mathbf{B}$ a ball around the origin, there is a finite $\bar{\alpha}$ such that $\Theta \subseteq \bar{\alpha} \mathbf{B}$ and then $\Theta$ is bounded. Necessity is proved by contradiction. Suppose that $\Theta$ is bounded and there is $\bar{x} \neq 0$ such that $\Phi_{\Theta}(\bar{x})<0$. Then, from positive homogeneity one has that $\Phi_{\Theta}(\alpha \bar{x})=\alpha \Phi_{\Theta}(\bar{x})<0$ for all $\alpha>0$. Then the whole ray passing through $\bar{x}$ is contained in $\Theta$, from (4.4), and hence $\Theta$ in unbounded, which contradicts the assumption. $\square$

COROLlaRY 4.7. For every bounded $A, B \in \mathscr{C}\left(\mathbb{R}^{n}\right)$ we have that $B \subseteq A$ if and only if $\Phi_{A}(x) \leq \Phi_{B}(x)$, for all $x \in \mathbb{R}^{n}$.

Proof. To prove necessity assume that $\Phi_{A}(x) \leq \Phi_{B}(x)$, for all $x \in \mathbb{R}^{n}$, and consider $x \in B$. From (4.4), $\Phi_{B}(x) \leq 1$ which implies $\Phi_{A}(x) \leq 1$ that is equivalent to $x \in A$. Thus $B \subseteq A$. Sufficiency is proved by contradiction. Suppose that $B \subseteq A$ and that there is $\bar{x} \neq 0$ such that $\Phi_{A}(\bar{x})>\Phi_{B}(\bar{x})$. From Proposition 4.6, $\Phi_{B}(\bar{x})>0$ and then there is $\alpha=\alpha(\bar{x})$ such that $\Phi_{B}(\alpha \bar{x})=1$, for homogeneity, which is equivalent to $\alpha \bar{x} \in B$. From the assumptions, $\Phi_{A}(\alpha \bar{x})>1$ that means $\alpha \bar{x} \notin A$ and then $B \nsubseteq A$, which contradicts the hypothesis.

We define the set of the indices of the active constraint of $\Theta \in \mathscr{C}\left(\mathbb{R}^{n}\right)$ for determining a function related to $\Theta$ which is connected, as shown in the following, to the contingent epiderivative of $\Phi_{\Theta}$. This is a computation-oriented tool for determining contractiveness conditions and induced polyhedral Lyapunov functions, possibly nonconvex.

DeFINITION 4.8. Given $\Theta \in \mathscr{C}\left(\mathbb{R}^{n}\right)$ denote with $H^{\Theta} \in \mathbb{R}^{n_{G} \times n}$ the matrix whose $n_{G}$ rows are given by the normals to the half-spaces determining $\Theta$ and

$$
I_{\Theta}(x)=\left\{i \in \mathbb{N}_{G}: H_{i}^{\Theta} x=\Phi_{\Theta}(x)\right\}
$$

and $\bar{I}_{\Theta}(x)=\mathbb{N}_{n_{G}} / I_{\Theta}(x)$. Given $x \in \mathbb{R}^{n}$, define $\Theta_{I}(x) \in \mathscr{C}\left(\mathbb{R}^{n}\right)$ the set obtained by keeping from the definition of $\Theta$ the constraints related to $j \in I_{\Theta}(x)$.

That is, $I_{\Theta}(x)$ denotes the indices of the active constraints of the level set of $\Phi_{\Theta}$ at $x$. As proved below, the set $\Theta_{I}(x)$ is related to a computationally suitable representation of the contingent epiderivative of $\Phi_{\Theta}$ at $x$.

EXAMPLE 4.9. Consider $A, B \in \mathscr{C}\left(\mathbb{R}^{n}\right)$ as in Example 4.4. Then if $\Theta=A \cap B$ and $\Omega=A \cup B$ we have

$$
\begin{aligned}
& \Phi_{\Theta_{I}(x)}(y)=\max \left\{\max \left\{H_{i}^{A} y: i \in \mathbb{N}_{a} \cap I_{\Theta}(x)\right\}, \min \left\{H_{j}^{B} y: j \in \mathbb{N}_{b} \cap I_{\Theta}(x)\right\}\right\}, \\
& \Phi_{\Omega_{I}(x)}(y)=\min \left\{\max \left\{H_{i}^{A} y: i \in \mathbb{N}_{a} \cap I_{\Omega}(x)\right\}, \min \left\{H_{j}^{B} y: j \in \mathbb{N}_{b} \cap I_{\Omega}(x)\right\}\right\} .
\end{aligned}
$$

Since $\Phi_{\Theta}$ for bounded $\Theta \in \mathscr{C}\left(\mathbb{R}^{n}\right)$ is continuous, which implies contingent epidifferentiability and lower semicontinuity, see [3], the result also applies to the case under analysis. The subsequent result, valid for any $\Theta \in \mathscr{C}\left(\mathbb{R}^{n}\right)$, is employed afterwards.

Proposition 4.10. Consider $\Theta \in \mathscr{C}\left(\mathbb{R}^{n}\right)$ with $H^{\Theta} \in \mathbb{R}^{n_{G} \times n}$. For every $x \in \mathbb{R}^{n}$ there exists a nonempty neighborhood $N(x)$ such that

$$
\Phi_{\Theta}(z)=\Phi_{\Theta_{I}(x)}(z),
$$


for every $z \in N(x)$.

Proof. First notice that condition (4.6) is equivalent to say that, for every $z \in N(x)$, there is no $j(z) \in \bar{I}_{\Theta}(x)$ such that $H_{j(z)} z=\Phi_{\Theta}(z)$. To prove the result it is sufficient to define $N(x)=x+\mathbf{B}^{n}(\varepsilon(x))$ with

$$
\varepsilon(x)=\min _{z \in \mathbb{R}^{n}}\left\{\|z-x\|_{2}: \exists j \in \bar{I}_{\Theta}(x), \text { s.t. }\left|H_{j} z-\Phi_{\Theta}(z)\right|=0\right\},
$$

and prove that $\varepsilon(x)>0$. In fact, suppose by contradiction that $\varepsilon(x)=0$. Then the point $z$ closest to $x$ and such that there is $j(z) \in \bar{I}_{\Theta}(x)$ for which $H_{j(z)} z=\Phi_{\Theta}(z)$, is $z=x$ and then $H_{j(z)} z=H_{j(z)} x=\Phi_{\Theta}(x)$. However, this would mean $j(z) \in I_{\Theta}(x)$, which contradicts $j(z) \in \bar{I}_{\Theta}(x)$, then it is absurd, which allows concluding the proof. $\square$

The corollary below follows from Proposition 4.10 and the fact that $\Phi_{\Theta}(x)=\Phi_{\Theta_{I}(x)}(x)$.

COROLLARY 4.11. Given $\Theta \in \mathscr{C}\left(\mathbb{R}^{n}\right)$, for every $x \in \mathbb{R}^{n}$ there exists a nonempty neighborhood of the origin $N^{0}(x)$ such that $\Phi_{\Theta}(x+v)=\Phi_{\Theta}(x)+\Phi_{\Theta_{I}(x)}(v)$, for every $v \in N^{0}(x)$.

The following proposition is employed in the main result proof.

PROPOSITION 4.12. Given $\Theta \in \mathscr{C}\left(\mathbb{R}^{n}\right)$, $\Phi_{\Theta_{I}(x)}(x+v)=\Phi_{\Theta_{I}(x)}(x)+\Phi_{\Theta_{I}(x)}(v)$ holds for every $x, v \in \mathbb{R}^{n}$.

Proof. The result is based on the fact that $\alpha+\max _{i}\left\{\beta^{i}\right\}=\max _{i}\left\{\alpha+\beta^{i}\right\}$ and $\alpha+$ $\min _{i}\left\{\beta^{i}\right\}=\min _{i}\left\{\alpha+\beta^{i}\right\}$ and the fact that $\Phi_{\Theta_{I}(x)}(x)=H_{i}^{\Theta} x$ for all $i \in I_{\Theta}(x)$.

Thus, the contingent epiderivative of $\Phi_{\Theta}$ at $x$ depends on $\Phi_{\Theta_{I}(x)}$ as proved below.

PROPOSITION 4.13. Consider the bounded set $\Theta \in \mathscr{C}\left(\mathbb{R}^{n}\right)$. The contingent epiderivative of $\Phi_{\Theta}$ at $x$ is given by $D_{\uparrow} \Phi_{\Theta}(x)(y)=\Phi_{\Theta_{I}(x)}(y)$, for all $y \in \mathbb{R}^{n}$.

Proof. As $h \rightarrow 0^{+}$, we can assume that $h v \in N^{0}(x)$ as in Corollary 4.11. From Corollary 4.11 and homogeneity of $\Phi_{\Theta}$ and $\Phi_{\Theta_{I}(x)}$, we have that

$$
\begin{aligned}
D_{\uparrow} \Phi_{\Theta}(x)(y) & =\liminf _{h \rightarrow 0^{+}, v \rightarrow y} \frac{\Phi_{\Theta}(x+h v)-\Phi_{\Theta}(x)}{h}=\liminf _{h \rightarrow 0^{+}, v \rightarrow y} \frac{\Phi_{\Theta}(x)+\Phi_{\Theta_{I}(x)}(h v)-\Phi_{\Theta}(x)}{h} \\
& =\liminf _{h \rightarrow 0^{+}, v \rightarrow y} \frac{h \Phi_{\Theta_{I}(x)}(v)}{h}=\liminf _{v \rightarrow y} \Phi_{\Theta_{I}(x)}(v) .
\end{aligned}
$$

Since $\Phi_{\Theta_{I}(x)}$ is continuous, the result follows. $\square$

LemmA 4.14. For every $x \in \mathbb{R}^{n}$ and $\Theta \in \mathscr{C}\left(\mathbb{R}^{n}\right)$, the set $\Gamma=\left\{v \in \mathbb{R}^{n}: \Phi_{\Theta_{I}(x)}(v) \leq 0\right\}$ is a closed cone.

Proof. From [3], the epigraph of the contingent epiderivative at $x$ is a closed cone. Then from Proposition 4.13, the epigraph of $\Phi_{\Theta_{I}(x)}(v)$ is a closed cone and so is $\Gamma$, since it is the intersection of two cones in $\mathbb{R}^{n+1}$, i.e. the epigraph of $\Phi_{\Theta_{I}(x)}(v)$ and the closed cone $\left\{y \in \mathbb{R}^{n+1}: y_{n+1}=0\right\}$.

Lemma 4.15. Given $y^{i} \in \mathbb{R}$ with $i \in \mathbb{N}_{y}, z \in \mathbb{R}$ and $\alpha \in \mathbb{R}$, then $\max \left\{\min _{i \in \mathbb{N}_{y}}\left\{y^{i}\right\}, z\right\} \leq \alpha$ if and only if there exists $j \in \mathbb{N}_{y}$ such that $\max \left\{y^{j}, z\right\} \leq \alpha$.

Proof. To prove necessity, suppose there is $j \in \mathbb{N}_{y}$ such that $\max \left\{y^{j}, z\right\} \leq \alpha$. From this and $\max \left\{\min _{i \in \mathbb{N}_{y}}\left\{y^{i}\right\}, z\right\} \leq \max \left\{y^{j}, z\right\}$ then $\max \left\{\min _{i \in \mathbb{N}_{y}}\left\{y^{i}\right\}, z\right\} \leq \alpha$. For sufficiency, suppose that $\max \left\{\min _{i \in \mathbb{N}_{y}}\left\{y^{i}\right\}, z\right\} \leq \alpha$ holds. The result is proved by posing $j=\arg \min _{i \in \mathbb{N}_{y}}\left\{y^{i}\right\}$ and noticing that $\min _{i \in \mathbb{N}_{y}}\left\{y^{i}\right\}=y^{j}$.

The lemma allows to formulate convex problems whose solution provides a characterization of the region in which $\Phi_{\Theta}$ decreases along the trajectory for an adequate $u(x)$.

EXAMPLE 4.16. Consider the bounded $\Theta \in \mathscr{C}\left(\mathbb{R}^{n}\right)$ as in Example 4.9. If the cardinality of $\mathbb{N}_{b} \cap I_{\Theta}(x)$ is greater than 1 , and since the minimum of linear functions may be nonconvex, 
then the function $\Phi_{\Theta_{I}(x)}$, defined in (4.5), might be nonconvex. From Lemma 4.15 we have that $\Phi_{\Theta_{I}(x)}(y) \leq 0$ if and only if there exists $j \in \mathbb{N}_{b} \cap I_{\Theta}(x)$ such that $\Phi_{\Theta_{I}^{i}(x)}(y) \leq 0$ where

$$
\Phi_{\Theta_{I}^{j}(x)}(y)=\max \left\{\max \left\{H_{i}^{A} y: i \in \mathbb{N}_{a} \cap I_{\Theta}(x)\right\}, H_{j}^{B} y\right\}=\max \left\{\left\{H_{i}^{A} y: i \in \mathbb{N}_{a} \cap I_{\Theta}(x)\right\}, H_{j}^{B} y\right\} .
$$

The result in Lemma 4.15 and its application in Example 4.16 are based on the fact that, given $A^{i} \subseteq \mathbb{R}^{n}$, with $i \in \mathbb{N}_{I}$ and $B \subseteq \mathbb{R}^{n},\left(\bigcup_{i \in \mathbb{N}_{I}} A^{i}\right) \cap B=\bigcup_{i \in \mathbb{N}_{I}}\left(A^{i} \cap B\right)$ holds. The relation with Lemma 4.15 stems from Definition 4.3, see also [36]. Thus, every $\Theta \in \mathscr{C}\left(\mathbb{R}^{n}\right)$ can be expressed as the finite union of convex sets, each obtained as the intersection of half-spaces $A=\left\{x \in \mathbb{R}^{n}: H x \leq 1\right\}$, see Definition 4.3.

Definition 4.17. Given $x \in \mathbb{R}^{n}$ and $\Theta \in \mathscr{C}\left(\mathbb{R}^{n}\right)$, define $\mathscr{K}_{j}(x) \subseteq I_{\Theta}(x)$, with $j \in \mathbb{N}_{J(x)}$ and $J(x) \in \mathbb{N}$, such that

$$
\Theta_{I}(x)=\bigcup_{j \in J(x)} \Theta_{\mathscr{K}_{j}}(x), \quad \text { with } \quad \Theta_{\mathscr{K}_{j}}(x)=\bigcap_{k \in \mathscr{K}_{j}(x)}\left\{y \in \mathbb{R}^{n}: H_{k}^{\Theta} y \leq 1\right\} .
$$

Therefore, one has

$$
\Phi_{\Theta_{I}(x)}(v)=\min _{j \in J(x)}\left\{\Phi_{\Theta_{\mathscr{K}_{j}}(x)}(v)\right\}=\min _{j \in J(x)}\left\{\max _{k \in \mathscr{K}_{j}(x)}\left\{H_{k}^{\Theta} v\right\}\right\} .
$$

REMARK 4.18. Notice that, from Lemma 4.14, the sets $\left\{v \in \mathbb{R}^{n}: \Phi_{\Theta_{\mathscr{K}_{j}}(x)}(v) \leq 0\right\}$ are convex cones.

5. Nonconvex polyhedral control Lyapunov functions and exponential stability. The results presented in this section provide a computation-oriented characterization of exponential stabilizability through Lyapunov functions for systems (2.1) such that Assumption 2.1 holds. The objective is to determine a condition for the function $\Phi_{\Theta}$ with $\Theta \in \mathscr{C}\left(\mathbb{R}^{n}\right)$ to be a local control Lyapunov function. For this, we provide computation-oriented necessary and sufficient conditions for the existence of $u(x) \in U(x)$ such that the contingent HamiltonJacobi equation (3.4) is satisfied in a region. This would imply that $\Phi_{\Theta}$ is a local control Lyapunov function and the system is locally asymptotically stabilizable where such conditions hold. Moreover, Theorem 3.4, applied with $\varphi(x)=\lambda x$ and $\lambda>0$, leads to conditions on the existence of a trajectory of system (2.1) that exponentially converges to the origin. For every $\Theta \in \mathscr{C}\left(\mathbb{R}^{n}\right)$, the function $\Phi_{\Theta}$ is contingently epidifferentiable and then Theorem 3.4 can be applied with $V(x)=\Phi_{\Theta}(x)$.

THEOREM 5.1. Let Assumption 2.1 hold and consider the nonempty bounded $\Theta \in$ $\mathscr{C}\left(\mathbb{R}^{n}\right)$. Then for every $x \in \mathbb{R}^{n}$ there exist $u(x) \in U(x)$ and $\mathscr{K}=\mathscr{K}_{j}(x)$, as in Definition 4.17, such that

$$
H_{k}^{\Theta}(g(x)+h(x) u(x)+\lambda x) \leq 0, \quad \forall k \in \mathscr{K},
$$

with $\lambda>0$, if and only if there exists $u(t) \in U(x(t))$ for $t \geq 0$, such that a solution to (2.1) satisfies

$$
\Phi_{\Theta}(x(t)) \leq \Phi_{\Theta}\left(x_{0}\right) e^{-\lambda t},
$$

for every $x_{0} \in \mathbb{R}^{n}$.

Proof. The first step is to prove that the existence of $u(x) \in U(x)$ such that

$$
\Phi_{\Theta_{I}(x)}(g(x)+h(x) u(x)+\lambda x) \leq 0,
$$


holds, is a necessary and sufficent condition for (5.2). Theorem 3.4, with $V=\Phi_{\Theta}$ and $\varphi(w)=$ $\lambda w$, and Proposition 4.13 imply that

$$
\inf _{u \in U(x)} \Phi_{\Theta_{I}(x)}(g(x)+h(x) u(x))+\lambda \Phi_{\Theta}(x) \leq 0, \quad \forall x \in \operatorname{dom}\left(\Phi_{\Theta}\right)=\mathbb{R}^{n},
$$

holds if and only if there exists $u(t) \in U(x(t))$, for $t \geq 0$, such that the solution to (2.1) fulfills (5.2), which corresponds to condition (3.3) for the current case. From Proposition 4.12 and homogeneity of $\Phi_{\Theta_{I}(x)}$ we have

$$
\begin{gathered}
\Phi_{\Theta_{I}(x)}(g(x)+h(x) u(x))+\lambda \Phi_{\Theta}(x)=\lambda\left(\Phi_{\Theta_{I}(x)}\left(\lambda^{-1} g(x)+\lambda^{-1} h(x) u(x)\right)+\Phi_{\Theta_{I}(x)}(x)\right)= \\
=\lambda\left(\Phi_{\Theta_{I}(x)}\left(\lambda^{-1} g(x)+\lambda^{-1} h(x) u(x)+x\right)\right)=\Phi_{\Theta_{I}(x)}(g(x)+h(x) u(x)+\lambda x) .
\end{gathered}
$$

Noticing that condition (5.4) is equivalent to claim the existence of $u(x) \in U(x)$ such that (5.3) is satisfied for all $x \in \mathbb{R}^{n}$, the first part of the result is proved.

From Definition 4.17, condition (5.3) is equivalent to the existence of $\mathscr{K}=\mathscr{K}_{i}(x)$ such that

$$
\Phi_{\Theta_{\mathscr{K}_{j}}(x)}(g(x)+h(x) u(x)+\lambda x) \leq 0,
$$

which is also equivalent to (5.1), and then the result follows. $\square$

One implication of Theorem 5.1 and Remark 4.18 is that $\Phi_{\Theta}(x)$ decreases at $x$ if and only if the vector $\dot{x}$ is contained, for appropriate $u(x)$, in one of the convex cones whose union determine the set $\left\{v \in \mathbb{R}^{n}: \Phi_{\Theta_{I}(x)}(v) \leq 0\right\}$. Notice that this would imply that the contingent epiderivative is negative along the trajectory.

REMARK 5.2. Theorem 5.1 yields a condition on $\Phi_{\Theta}$ to be a global control Lyapunov function for the system (2.1) under Assumption 2.1. The fact that the effective domain of $\Phi_{\Theta}$ is $\mathbb{R}^{n}$ for every $\Theta \in \mathscr{C}\left(\mathbb{R}^{n}\right)$ is implicitly used. Local Lyapunov functions can be defined modifying adequately the function by defining it $+\infty$ outside of the domain of attraction.

Then, we have proved that (5.1) with $\mathscr{K}=\mathscr{K}_{j}(x)$, satisfied by $u(x) \in U(x)$, is a necessary and sufficient condition for $\Phi_{\Theta}(x)$ with bounded $\Theta \in \mathscr{C}\left(\mathbb{R}^{n}\right)$, to be a local control Lyapunov function for system (2.1). Furthermore, the value of $\Phi_{\Theta}(x(t))$ is bounded above by an exponentially decreasing function whose time constant is $\lambda$.

REMARK 5.3. The results presented above concerning $\Phi_{\Theta}$ with bounded $\Theta \in \mathscr{C}\left(\mathbb{R}^{n}\right)$ as potential control Lyapunov function, as well as the characterization of its contingent epiderivative at $x$ through $\Phi_{\Theta_{I}(x)}$, are valid for more general functions, see [3, 15]. Nonetheless, the function $\Phi_{\Theta}$ for $\Theta \in \mathscr{C}\left(\mathbb{R}^{n}\right)$ is particularly suitable for computational purposes, as shown below.

6. Computational issues. From Theorem 5.1, the satisfaction of condition (5.1), where $\mathscr{K}=\mathscr{K}_{j}(x)$ identifies a convex cone determining the contingent epiderivative of $\Phi_{\Theta}(x)$, is necessary and sufficient for the function induced by $\Theta$ to be decreasing at $x$. Then, from the computational point of view, the first problem is to characterize the region where, given a convex cone, the condition (5.1) can be satisfied. This issue is dealt with in Section 6.1. After that, some aspects on the computation of the extreme points of the feasible region of the dual problem are considered in Section 6.2. The interest resides in the fact that these points completely characterize the region in which condition (5.1) is satisfied, and then where the candidate function has a decreasing rate greater than $\lambda$ for an appropriate $u(x)$. In Section 6.3, it will be shown that these extremes determine also such a control input. Finally, the direct implications for the original problem with potentially nonconvex contingent epiderivative are presented in Section 6.4. 
6.1. Exact region of viability for convex cones. As illustrated in Section 4, the contingent epiderivative of $\Phi_{\Theta}(x)$ is determined for every $x \in \mathbb{R}^{n}$ by the union of convex cones defined by the active constraints, see Definition 4.17.

DEFINITION 6.1. Given the set $\mathscr{K} \subseteq \mathbb{N}_{n_{h}}$ define

$$
R_{\mathscr{K}}=\left\{x \in \mathbb{R}^{n}: k \in \mathscr{K} \Rightarrow H_{k}^{\Theta} x=\Phi_{\Theta}(x)\right\},
$$

and $\Theta_{\mathscr{K}}=\left\{x \in \mathbb{R}^{n}: H_{k}^{\Theta} x \leq 1, \forall k \in \mathscr{K}\right\}$.

That is, $R_{\mathscr{K}}$ is the region in which the constraints indexed by $\mathscr{K}$ are active, possibly not exclusively.

REMARK 6.2. Notice that $R_{\mathscr{K}}$ might be empty and that the union of $R_{\mathscr{K}}$ for all $\mathscr{K} \subseteq \mathbb{N}_{n_{h}}$ is $\mathbb{R}^{n}$. Moreover $\Theta_{\mathscr{K}}$ is a convex cone containing the origin in its interior and $H_{k}^{\Theta} x=\Phi_{\Theta_{\mathscr{K}}}(x)$ for all $k \in \mathscr{K}$ and every $x \in \mathbb{R}_{\mathscr{K}}$.

We consider hereafter the region determined by a generic $\mathscr{K} \subseteq \mathbb{N}_{n_{h}}$. Given $\mathscr{K} \subseteq \mathbb{N}_{n_{h}}$ and $x \in R_{\mathscr{K}}$, as in (6.1), we define the following optimization problem, whose solution characterizes the states $x \in R_{\mathscr{K}}$ at which condition (5.1) holds.

Definition 6.3 (Primal problem). Let Assumption 2.1 hold. Given $\mathscr{K} \subseteq \mathbb{N}_{h_{h}}$ and $x \in R_{\mathscr{K}}$, define the following optimization problem:

$$
\begin{aligned}
& \alpha_{\mathscr{K}}^{\lambda, *}(x)=\min _{\alpha, u} \alpha, \\
& \text { s.t. } H_{i}^{\Theta} x \leq \alpha, \quad \forall i \in \mathscr{K} \text {, } \\
& H_{k}^{\Theta}(g(x)+h(x) u+\lambda x)+H_{k}^{\Theta} x-\alpha \leq 0, \quad \forall k \in \mathscr{K}, \\
& M_{j} u \leq N_{j}(x), \quad \forall j \in \mathbb{N}_{n_{u}} \text {, } \\
& \alpha \geq 0 \text {. }
\end{aligned}
$$

A computation-oriented necessary and sufficient condition for (5.1) to hold at $x \in R_{\mathscr{K}}$ stems from the following proposition.

Proposition 6.4. Given $\mathscr{K} \subseteq \mathbb{N}_{n_{h}}$ and $x \in R_{\mathscr{K}}$, the optimal solution $\alpha_{\mathscr{K}}^{\lambda, *}(x)$ to the primal problem (6.2) is such that $\Phi_{\Theta_{\mathscr{K}}}(x)=\alpha_{\mathscr{K}}^{\lambda, *}(x)$ if and only if there exists $u \in U(x)$ such that condition (5.1) holds at $x$. Furthermore, $\Phi_{\Theta_{\mathscr{K}}}(x)<\alpha_{\mathscr{K}}^{\lambda, *}(x)$ if and only if condition (5.1) is not satisfied at $x$ for any $u \in U(x)$.

Proof. Suppose that condition (5.1) holds at $x \in R_{\mathscr{K}}$ for a $u(x) \in U(x)$ and consider such a $u(x)$ that satisfies the third constraint of (6.2). Then the second set of constraints in (6.2) is satisfied by every $\alpha \geq 0$ such that $\Phi_{\Theta_{\mathscr{K}}}(x)=H_{k}^{\Theta} x \leq \alpha$, as well as the first set of constraints. Since the optimization problem minimizes $\alpha$, its optimal value is $\Phi_{\Theta_{\mathscr{K}}}(x)$, that proves the necessity of the first part of the proposition. Concerning the sufficiency, suppose that the optimal value of problem (6.2) is given by $\Phi_{\Theta_{\mathscr{K}}}(x)$, i.e. suppose that $\alpha_{\mathscr{K}}^{\lambda, *}(x)=\Phi_{\Theta_{\mathscr{K}}}(x)$. Then the first set of constraints is satisfied by definition, in fact $H_{i}^{\Theta} x=\Phi_{\Theta \mathscr{K}}(x)=\alpha_{\mathscr{K}}^{\lambda, *}(x)$, for all $i \in \mathscr{K}$. Moreover, it follows that $H_{k}^{\Theta} x=\alpha_{\mathscr{K}}^{\lambda, *}(x)$ for all $k \in \mathscr{K}$ and then the second set of constraints in (6.2) becomes the condition (5.1). Hence, the solution $\alpha_{\mathscr{K}}^{\lambda, *}(x)$ is equal to the $\Phi_{\Theta_{\mathscr{K}}}(x)$ if and only if the condition (5.1) is satisfied at $x$.

Furthermore we have that $\Phi_{\Theta_{\mathscr{K}}}(x) \leq \alpha_{\mathscr{K}}^{\lambda, *}(x)$. Indeed, the value of $\Phi_{\Theta_{\mathscr{K}}}(x)$ would be obtained as the optimum by removing the second set of constraints and solving the optimization problem. However, since this problem has a larger feasible region, its optimum is smaller than or equal to $\alpha_{\mathscr{K}}^{\lambda, *}(x)$. Then $\alpha_{\mathscr{K}}^{\lambda, *}(x)$ cannot be smaller than $\Phi_{\Theta}(x)$. This and the fact that $\Phi_{\Theta_{\mathscr{K}}}(x)=\alpha_{\mathscr{K}}^{\lambda, *}(x)$ if and only if condition (5.1) is satisfied at $x$, imply that the optimal value is greater than $\Phi_{\Theta}(x)$ if and only if (5.1) is not fulfilled at $x$. $\mathrm{Y}$ 
Conditions for characterizing the regions of the state space in which (5.1) is satisfied are provided below. Notice that (6.2) is a linear optimization problem in $\alpha$ and $u$ with feasible solutions. Then, strong duality holds in our case, see [11,6]. Applying classical results from duality for convex optimization problems we obtain the following problem, dual of (6.2).

Definition 6.5 (Dual problem). Let Assumption 2.1 hold. Given $\mathscr{K} \subseteq \mathbb{N}_{h_{h}}$ and $x \in$ $R_{\mathscr{K}}$, the dual of the linear optimization problem (6.2) is

$$
\begin{aligned}
L_{\mathscr{K}}^{\lambda, *}(x)=\max _{\beta, \delta, \sigma} & L_{\mathscr{K}}^{\lambda}(\beta, \delta, \sigma ; x), \\
\text { s.t. } \quad & \sum_{i \in \mathscr{K}} \beta_{i}+\sum_{k \in \mathscr{K}} \delta_{k} \leq 1, \\
& \sum_{k \in \mathscr{K}} \delta_{k} H_{k}^{\Theta} h(x)+\sum_{j=1}^{n_{u}} \sigma_{j} M_{j}=0, \\
& \beta \geq 0, \quad \delta \geq 0, \quad \sigma \geq 0,
\end{aligned}
$$

with

$$
L_{\mathscr{K}}^{\lambda}(\beta, \delta, \sigma ; x)=\sum_{i \in \mathscr{K}} \beta_{i} H_{i}^{\Theta} x+\sum_{k \in \mathscr{K}} \delta_{k} H_{k}^{\Theta} g(x)+\sum_{k \in \mathscr{K}} \delta_{k}(\lambda+1) H_{k}^{\Theta} x-\sum_{j=1}^{n_{u}} \sigma_{j} N_{j}(x) .
$$

The optimal value of the dual problem is such that $L_{\mathscr{K}}^{\lambda}(\beta, \delta, \sigma ; x) \leq L_{\mathscr{K}}^{\lambda, *}(x)$ for all feasible $(\beta, \delta, \sigma)$. We used the notation $(\beta, \delta, \sigma)$ in spite of $(\beta(x), \delta(x), \sigma(x))$ for simplicity. Hence $L_{\mathscr{K}}^{\lambda, *}(x)$ is the maximal lower bound of $\alpha_{\mathscr{K}}^{\lambda, *}(x)$ and, from strong duality, $L_{\mathscr{K}}^{\lambda, *}(x)=\alpha_{\mathscr{K}}^{\lambda, *}(x)$. Then, $L_{\mathscr{K}}^{\lambda}(\beta, \delta, \sigma ; x) \leq \alpha_{\mathscr{K}}^{\lambda, *}(x)$ for any feasible solution to (6.3).

PROPOSITION 6.6. A necessary and sufficient condition for (5.1) to hold at $x \in R_{\mathscr{K}}$ is

$$
L_{\mathscr{K}}^{\lambda, *}(x) \leq H_{k}^{\Theta} x, \quad \forall k \in \mathscr{K},
$$

with $L_{\mathscr{K}}^{\lambda, *}(x)$ as in (6.3), or, equivalently, $L_{\mathscr{K}}^{\lambda}(\beta, \delta, \sigma ; x) \leq H_{k}^{\Theta} x$ for every $(\beta, \delta, \sigma)$ feasible solution to (6.3).

Proof. From strong duality, $L_{\mathscr{K}}^{\lambda, *}(x)=\alpha_{\mathscr{K}}^{\lambda, *}(x)$. This implies $H_{k}^{\Theta} x=\Phi_{\Theta_{\mathscr{K}}}(x) \leq L_{\mathscr{K}}^{\lambda, *}(x)$ for all $k \in \mathscr{K}$, as proved for Proposition 6.4, and then $\Phi_{\Theta_{\mathscr{K}}}(x)=L_{\mathscr{K}}^{\lambda, *}(x)$ if and only if (5.1) holds at $x \in R_{\mathscr{K}}$. Then (5.1) holds at $x \in R_{\mathscr{K}}$ if and only if (6.5) is satisfied. $\square$

Posing the condition for (5.1) to hold in the inequality form (6.5), rather than as equality constraint $L_{\mathscr{K}}^{\lambda, *}(x)=H_{k}^{\Theta} x$ for all $k \in \mathscr{K}$, leads to convex optimization problems under adequate assumptions on $U(x)$. Consider the dual problem (6.3). Given $\mathscr{K} \subseteq \mathbb{N}_{n_{h}}$ and $x \in R_{\mathscr{K}}$, the problem of checking if (5.1) holds reduces to the maximization of a linear function over a polyhedral set in the space of variables $\beta, \delta$ and $\sigma$. Then, the maximum is attained at an extreme point or the problem is unbounded. Since the primal optimum exists and is bounded, the analysis can be reduced to the extreme points of the dual problem feasibility region.

PROPERTY 6.7. The optimal value of the dual problem (6.3) is attained at an extreme point of the feasibility region.

Proof. Since the origin is an extreme point of the feasibility region of the dual problem (6.3), which is bounded above by the primal optimal value, the result is implied by the Fundamental Theorem of Linear Programming, see [6].

Based on Proposition 6.6 and Property 6.7, a computation-oriented necessary and sufficient condition for (5.1) to hold al $x \in R_{\mathscr{K}}$ is presented below.

THEOREM 6.8. Given $\mathscr{K} \subseteq \mathbb{N}_{n_{h}}$, denote with $\left(\beta^{p}, \delta^{p}, \sigma^{p}\right)$ the $p$-th extreme point of the feasibility region of the dual problem (6.3), with $p \in \mathbb{N}_{n_{v}}$. The subset of $x \in R_{\mathscr{K}}$ at which (5.1) 
holds is given by

$$
\mathscr{V}_{\mathscr{K}}^{\lambda}=\bigcap_{p \in \mathbb{N}_{n_{v}}}\left\{x \in \mathbb{R}^{n}: L_{\mathscr{K}}^{\lambda}\left(\beta^{p}, \delta^{p}, \sigma^{p} ; x\right) \leq H_{k}^{\Theta} x, \quad \forall k \in \mathscr{K}\right\},
$$

with $L_{\mathscr{K}}^{\lambda}$ as in (6.4). The subset of $x \in R_{\mathscr{K}}$ at which (5.1) does not hold is

$$
\overline{\mathscr{V}}_{\mathscr{K}}^{\lambda}=\bigcup_{p \in \mathbb{N}_{n_{v}}}\left\{x \in \mathbb{R}^{n}: L_{\mathscr{K}}^{\lambda}\left(\beta^{p}, \delta^{p}, \sigma^{p} ; x\right)>H_{k}^{\Theta} x, \quad \forall k \in \mathscr{K}\right\} .
$$

Proof. Property 6.7 implies that for all $x \in R_{\mathscr{K}}$ there exists $p^{*}=p^{*}(x) \in \mathbb{N}_{n_{v}}$ such that

$$
L_{\mathscr{K}}^{\lambda}(\beta, \delta, \sigma ; x) \leq L_{\mathscr{K}}^{\lambda}\left(\beta^{p^{*}}, \delta^{p^{*}}, \sigma^{p^{*}} ; x\right)=L_{\mathscr{K}}^{\lambda, *}(x),
$$

for feasible $(\beta, \delta, \sigma)$. This and Proposition 6.6 prove the first claim. Similar considerations and the fact that $\Phi_{\Theta_{\mathscr{K}}}(x)=H_{k}^{\Theta} x$ for all $k \in \mathscr{K}$ for every $x \in R_{\mathscr{K}}$, prove the second claim. $\square$

The set $\mathscr{V}_{\mathscr{K}}^{\lambda}$, given by the intersection of subsets of the state space, is the exact region of all $x \in R_{\mathscr{K}}$ where condition (5.1) is satisfied by an adequate $u(x) \in U(x)$. The only optimization problem to solve for characterizing $\mathscr{V}_{\mathscr{K}}^{\lambda}$ concerns the computation of the extremes of the dual problem, the computation of $u(x)$ being not required.

6.2. Computation of the extremes of the dual feasible set. As shown above, to test if (5.1) holds in the region $R_{\mathscr{K}}$, with $\mathscr{K} \subseteq \mathbb{N}_{n_{h}}$, requires to compute the extreme points of the dual problem feasibility region. The formal definition of extreme point follows, see [6].

DEFINITION 6.9. Given a convex set $C, x \in C$ is an extreme point of $C$ if there are not two points $y \in C$ and $z \in C$ and a scalar $\lambda \in(0,1)$ such that $x=\lambda y+(1-\lambda) z$.

Obtaining the extreme points of a polyhedron is rather computationally demanding in general. We prove below that in this case the computation is less demanding.

THEOREM 6.10. Given $\mathscr{K} \subseteq \mathbb{N}_{n_{h}}$, with $n_{i}$ cardinality of $\mathscr{K}$, any extreme $(\beta, \delta, \sigma) \in$ $\mathbb{R}^{n_{i}+n_{i}+n_{u}}$ of the feasible region of (6.3) is either such that $\delta=0, \sigma=0$ and all the entries of $\beta$ are 0 except one equal to 1 , or such that $\beta=0$ and $(\delta, \sigma)$ is an extreme point of

$$
\left\{\begin{array}{l}
\sum_{k \in \mathscr{K}} \delta_{i} H_{i}^{\Theta} h(x)+\sum_{j=1}^{n_{u}} \sigma_{j} M_{j}=0, \\
\sum_{k \in \mathscr{K}} \delta_{i}=1 \\
\delta \geq 0, \quad \sigma \geq 0
\end{array}\right.
$$

or it is the origin of $\mathbb{R}^{n_{i}+n_{i}+n_{u}}$.

Proof. First denote the feasible set of (6.3) as $F_{\mathscr{K}}$ and define $\gamma=\sum_{k \in \mathscr{K}} \delta_{i}$. The proof of the theorem is substantially based on demonstrating that for every extreme point $(\beta, \delta, \sigma)$ of $F_{\mathscr{K}}$, either $\gamma=0$ or $\gamma=1$. Indeed, if this is true, the values of $\beta$ at the extremes of $F_{\mathscr{K}}$ can be decoupled by the other dual variables. Notice that from (6.3) we have that, depending on whether $\gamma=0$ or $\gamma=1, \beta$ at the extremes of $F_{\mathscr{K}}$ are the extremes of

$$
\left\{\begin{array} { l } 
{ \sum _ { i \in \mathscr { K } } \beta _ { i } \leq 1 , } \\
{ \beta \geq 0 , \quad \gamma = 0 , }
\end{array} \quad \text { or } \quad \left\{\begin{array}{l}
\sum_{i \in \mathscr{K}} \beta_{i} \leq 0, \\
\beta \geq 0, \quad \gamma=1 .
\end{array}\right.\right.
$$

Then, for every extreme such that $\gamma=0, \beta$ is an extreme of the unit simplex in $\mathbb{R}^{n_{i}}$, that is either the origin or a vector whose entries are all 0 except one equal to 1 . If $\gamma=1$ at an extreme of $F_{\mathscr{K}}$, then $\beta=0$. We prove that if $F_{\mathscr{K}}$ has an extreme point such that $\gamma>0$, 
then it must be such that $\gamma=1$. We prove it by contradiction. Suppose that there exists an extreme point of $F_{\mathscr{K}}$ such that $\gamma>0$ and $\gamma \neq 1$. Clearly, we must have $\gamma<1$ (besides $\delta_{i} \geq 0$ for every $i \in \mathscr{K}$ ) otherwise the first constraint in (6.3) would not be satisfied by any $\beta \geq 0$. Then denote $\bar{v}=(\bar{\beta}, \bar{\delta}, \bar{\sigma})$ the extreme point of $F_{\mathscr{K}}$ with $\sum_{k \in \mathscr{K}} \bar{\delta}_{i}=\bar{\gamma}=\gamma \in(0,1)$ and consider $\hat{v}=\left(0, \bar{\gamma}^{-1} \bar{\delta}, \bar{\gamma}^{-1} \bar{\sigma}\right)$ and $\check{v}=\left((1-\bar{\gamma})^{-1} \bar{\beta}, 0,0\right)$. First notice that $\bar{v}$ is strictly included in the segment whose endpoints are $\hat{v}$ and $\breve{v}$. In fact $\bar{\gamma} \in(0,1)$ and $\bar{\gamma} \hat{v}+(1-\bar{\gamma}) \check{v}=$ $(0, \bar{\delta}, \bar{\sigma})+(\bar{\beta}, 0,0)=\bar{v}$. Moreover, both $\hat{v}$ and $\breve{v}$ are feasible points of the dual problem (6.3). Indeed, from the fact that $\bar{v}$ satisfies the constraints in (6.3), we have for $\hat{v}$ :

$$
\left\{\begin{array}{l}
\sum_{i \in \mathscr{K}} 0+\bar{\gamma}^{-1} \sum_{k \in \mathscr{K}} \bar{\delta}_{i}=1, \\
\bar{\gamma}^{-1} \sum_{k \in \mathscr{K}} \bar{\delta}_{i} H_{i}^{\Theta} h(x)+\bar{\gamma}^{-1} \sum_{j=1}^{n_{u}} \bar{\sigma}_{j} M_{j}=0,
\end{array}\right.
$$

and considering $\check{v}$ we have

$$
\left\{\begin{array}{l}
\sum_{i \in \mathscr{K}} \frac{\bar{\beta}_{i}}{1-\gamma}+0 \leq 1 \quad \Leftrightarrow \quad \sum_{i \in \mathscr{K}} \bar{\beta}_{i}+\sum_{i \in \mathscr{K}} \bar{\delta}_{i} \leq 1, \\
0+\sum_{j=1}^{n_{u}} 0=0 .
\end{array}\right.
$$

Thus, $\bar{v}$ is strictly contained in the segment belonging to the feasible region, then it is not an extreme, see Definition 6.9, and this contradicts the assumption. Hence for every extreme point of $F_{\mathscr{K}}$, either $\gamma=0$ (which is equivalent to $\delta_{i}=0$ for every $k \in \mathscr{K}$ ) or $\gamma=1$.

Consider the case of extreme points $(\beta, \delta, \sigma)$ such that $\gamma=0$. Then, for what claimed above, $\beta$ is an extreme of the unit simplex in $\mathbb{R}^{n_{i}}$. We prove that, in this case, the only possible value of $\sigma$ is 0 . Suppose by contradiction that $(\beta, 0, \sigma)$ is an extreme and $\sigma \neq 0$. Then $\sigma \geq 0$ and there is $i \in \mathbb{N}_{n_{u}}$ such that $\sigma_{i}>0$ for which $\sum_{j=1}^{n_{u}} \sigma_{j} M_{j}=0$. Clearly $\sum_{j=1}^{n_{u}} \alpha \sigma_{j} M_{j}=0$ for every $\alpha>0$, which means that $(\beta, 0, \alpha \sigma) \in F_{\mathscr{K}}$ for all $\alpha>0$. Hence for every $\alpha_{1}, \alpha_{2}>0$ with $0<\alpha_{1}<1<\alpha_{2}$, we have that $(\beta, 0, \sigma)$ is strictly contained in the segment whose endpoints are $\left(\beta, 0, \alpha_{1} \sigma\right)$ and $\left(\beta, 0, \alpha_{2} \sigma\right)$, which are in $F_{\mathscr{K}}$. Then $(\beta, 0, \sigma)$ is not an extreme point of $F_{\mathscr{K}}$ which contradicts the assumption. Hence, every extreme point of $F_{\mathscr{K}}$ with $\gamma=0$ is such that $\beta$ is an extreme of the unit simplex in $\mathbb{R}^{n_{i}}$ and $\sigma=0$.

Finally, notice that if $\gamma=1$ then, necessarily, $\beta=0$, see (6.9), and constraints in (6.3) reduce to (6.8). Then $(\delta, \sigma)$ must be an extreme point of (6.8).

From Theorem 6.10, the computation of the extreme points of the feasible set of (6.3), polyhedron in $\mathbb{R}^{n_{i}+n_{i}+n_{u}}$, is reduced to the obtainment of the extreme points of the polyhedron in $\mathbb{R}^{n_{i}+n_{u}}$ given by (6.8), with, we recall, $n_{i}$ cardinality of $\mathscr{K}$ and $n_{u}$ number of linear inequalities defining the set-valued map $U$.

6.3. Optimal control input computation for convex cones. We have shown how to determine whether and where condition (5.1) holds. Recall that the satisfaction of (5.1) implies the existence of a local control law such that the value of the set-induced Lyapunov function decreases along one trajectory of the closed-loop system. We consider the problem of the computation of such a control action, possibly optimal with respect to a performance measure. One possibility is to look at the control law such that the decreasing rate of the function $\Phi_{\Theta \mathscr{K}}$ is maximized.

Every region $R_{\mathscr{K}}$, as defined in (6.1), is taken into account independently. This leads to a discontinuous optimal control law. Given a $x \in \mathscr{V}_{\mathscr{K}}^{\lambda}$, that is an $x$ at which the condition (5.1) is satisfied by some admissible inputs, we provide a method for characterizing such inputs.

Proposition 6.11. Given the $\Theta \in \mathscr{C}\left(\mathbb{R}^{n}\right)$ and $\mathscr{K} \subseteq \mathbb{N}_{n_{h}}$, the condition (5.1) holds at $x \in \mathscr{V}_{\mathscr{K}}^{\lambda}$ for an adequate $u$ if and only if there exist $u^{*}=u^{*}(\mathscr{K}, x) \in \mathbb{R}^{n_{u}},\left(\delta^{*}, \sigma^{*}\right) \in \mathbb{R}^{n_{i}+n_{u}}$ 
and $\mu^{*}=\mu^{*}(\mathscr{K}, x) \in \mathbb{R}$ satisfying

$$
\begin{cases}M_{j} u^{*}-N_{j}(x) \leq 0, & \forall j \in \mathbb{N}_{n_{u}}, \\ H_{k}^{\Theta} g(x)+H_{k}^{\Theta} h(x) u^{*} \leq \mu^{*}, & \forall k \in \mathscr{K} \\ \delta^{*} \geq 0, \quad \sigma^{*} \geq 0, & \forall j \in \mathbb{N}_{n_{u}}, \\ \sigma_{j}^{*}\left(M_{j} u^{*}-N_{j}(x)\right)=0, & \forall k \in \mathscr{K} \\ \delta_{k}^{*}\left(H_{k}^{\Theta} g(x)+H_{k}^{\Theta} h(x) u^{*}-\mu^{*}\right)=0, & \\ \sum_{k \in \mathscr{K}} \delta_{k}^{*}=1, & \\ \sum_{k \in \mathscr{K}} \delta_{k}^{*} H_{k}^{\Theta} h(x)+\sum_{j=1}^{n_{u}} \sigma_{j}^{*} M_{j}=0 . & \end{cases}
$$

Proof. Condition (5.1) holds if and only if there exists $u \in U(x)$ such that

$$
\begin{array}{r}
-\lambda \Phi_{\Theta \mathscr{K}}(x) \geq \min _{u} \max _{k \in \mathscr{K}}\left\{H_{k}^{\Theta} g(x)+H_{k}^{\Theta} h(x) u\right\} \\
\text { s.t. } \quad M_{j} u \leq N_{j}(x), \quad \forall j \in \mathbb{N}_{n_{u}},
\end{array}
$$

as shown in the proof of Theorem 5.1. From $x \in \mathscr{V}_{\mathscr{K}}^{\lambda}$, condition (5.1) is satisfied for the optimizer $u$ of (6.11). Notice that the optimization problem in (6.11) is equivalent to

$$
\begin{array}{lll}
\min _{u, \mu} & \mu \\
\text { s.t. } & H_{k}^{\Theta} g(x)+H_{k}^{\Theta} h(x) u \leq \mu, \quad \forall k \in \mathscr{K}, \\
& M_{j} u \leq N_{j}(x), & \forall j \in \mathbb{N}_{n_{u}},
\end{array}
$$

which is a linear programming problem in $u$ and $\mu$, parameterized in $x$. Since the KarushKuhn-Tacker (KKT) conditions, given by (6.10), are necessary and sufficient for the primaldual optimality in this case, see [11], then $u^{*}$, solution to (6.10), leads to satisfaction of (5.1).

प

From the practical point of view, the control input $u^{*}(\mathscr{K}, x)$ within the generic region $\mathscr{V}_{\mathscr{K}}^{\lambda}$ can be obtained by finding the extremes of (6.8). Indeed, the dual problem of (6.12) is given by

$$
\begin{array}{ll}
\max _{\sigma, \delta} & \sum_{k \in \mathscr{K}} H_{k}^{\Theta} g(x)-\sum_{j=1}^{n_{u}} \sigma_{j} N_{j}(x), \\
\text { s.t. } & \sum_{k \in \mathscr{K}} \delta_{k} H_{k}^{\Theta} h(x)+\sum_{j=1}^{n_{u}} \sigma_{j} M_{j}=0, \\
& \sum_{k \in \mathscr{K}} \delta_{k}=1, \\
& \delta \geq 0, \quad \sigma \geq 0,
\end{array}
$$

and then $\left(\delta^{*}, \sigma^{*}\right)$ is a feasible solution to the dual problem (6.13), an optimal one, in fact. Since the optimum of a linear optimization problem is attained at an extreme point, then the extremes computed to determine the domain $\mathscr{V}_{\mathscr{K}}^{\lambda}$ provide also the control $u^{*}=u^{*}(\mathscr{K}, x)$. In practice, for every extreme of (6.8), one can check whether the other constraints in (6.10) admit a solution with respect to $u^{*}=u^{*}(\mathscr{K}, x)$ and $\mu^{*}=\mu^{*}(\mathscr{K}, x)$. This happens if and only if $u^{*}$ and $\mu^{*}$ are optimal for the primal (6.12). Moreover, $\sigma^{*}$ are such that the optimal control satisfies

$$
M_{j} u^{*}=N_{j}(x), \quad \forall \sigma_{j}^{*}>0 .
$$

Furthermore, if $\delta_{i}^{*}>0$ then the constraint $\min _{u} \max _{k \in \mathscr{K}}\left\{H_{k}^{\Theta} g(x)+H_{k}^{\Theta} h(x) u\right\} \geq H_{i}^{\Theta} g(x)+H_{i}^{\Theta} h(x) u^{*}$, is active since $H_{i}^{\Theta} g(x)+H_{i}^{\Theta} h(x) u^{*}=\mu^{*}$. 
Besides being a condition for (5.1) to hold, and then for $\Phi_{\Theta_{\mathscr{K}}}(x)$ to be decreasing, (6.10) provides the potential control inputs $u^{*}$ which ensure the maximal decreasing rate along a trajectory.

COROLlary 6.12. Given $\Theta \in \mathscr{C}\left(\mathbb{R}^{n}\right)$ and $\mathscr{K} \subseteq \mathbb{N}_{n_{h}}$, any $u^{*}=u^{*}(\mathscr{K})$ solution to (6.10) is optimal with respect to

$$
\inf _{u \in U(x)} \Phi_{\Theta \mathscr{K}}(g(x)+h(x) u)
$$

and satisfies (5.1) if $x \in \mathscr{V}_{\mathscr{K}}^{\lambda}$.

Proof. The results following directly from Proposition 6.11 and the considerations given in its proof.

6.4. Exact region and optimal control for nonconvex epiderivative. Finally, the characterization of the exact regions where $\Phi_{\Theta}(x)$, with $\Theta \in \mathscr{C}\left(\mathbb{R}^{n}\right)$, decreases and the computation of the control input that maximizes the decreasing rate can be given.

Proposition 6.13. Let Assumption 2.1 hold and the nonempty bounded $\Theta \in \mathscr{C}\left(\mathbb{R}^{n}\right)$, consider $u^{*}\left(\mathscr{K}_{j}(x), x\right)$ and $\mu^{*}\left(\mathscr{K}_{j}(x), x\right)$ solutions to $(6.10)$ with $\mathscr{K}=\mathscr{K}_{j}(x)$, for all $j \in J(x)$ as in Definition 4.17. The input $u^{*}(x)=u^{*}\left(\mathscr{K}_{j^{*}}(x), x\right)$ such that

$$
j^{*}=\arg \min _{j \in J(x)}\left\{\mu^{*}\left(\mathscr{K}_{j}(x), x\right)\right\},
$$

is the optimizer of

$$
\inf _{u \in U(x)} D_{\uparrow} \Phi_{\Theta}(g(x)+h(x) u),
$$

and satisfies (5.1) if and only if $x \in \mathscr{V}_{\mathscr{K}_{j^{*}}(x)}^{\lambda}$.

Proof. From Proposition 6.11 and Corollary 6.12, $u^{*}\left(\mathscr{K}_{j}(x), x\right)$ and $\mu^{*}\left(\mathscr{K}_{j}(x), x\right)$ are optimizer and optimum of (6.15), with $\mathscr{K}=\mathscr{K}_{j}(x)$. From Definition 4.17 and Theorem 5.1 we have that (6.17) is equivalent to

$\inf _{u \in U(x)} \min _{j \in J(x)} \Phi_{\mathscr{K}_{j}(x)}(g(x)+h(x) u)=\min _{j \in J(x)} \inf _{u \in U(x)} \Phi_{\mathscr{K}_{j}(x)}(g(x)+h(x) u)=\min _{j \in J(x)}\left\{\mu^{*}\left(\mathscr{K}_{j}(x), x\right)\right\}$,

and thus (5.1) holds if and only if $x \in \mathscr{V}_{\mathscr{K}_{j}^{*}(x)}^{\lambda}$, see Proposition 6.11.

From the computational point of view, Proposition 6.13 means that $\Phi_{\Theta}(x)$ can be decreasing faster than $\lambda \Phi_{\Theta}(x)$ at $x$ if and only if $x \in \mathscr{V}_{\mathscr{K}_{j}(x)}^{\lambda}$ for at least one value of $j \in J(x)$. Moreover, the optimal control is determined by the KKT conditions. Thus, computing $\mathscr{V}_{\mathscr{K}}^{\lambda}$ for all possible $\mathscr{K} \subseteq \mathbb{N}_{n_{h}}$ would provide the exact characterization of the region where $\Phi_{\Theta}(x)$ decreases and also the optimal control.

7. Brockett's example. Consider the nonlinear system, called nonholonomic (or Brockett's) integrator [12], whose dynamics is

$$
\left\{\begin{array}{l}
\dot{x}_{1}=u_{1}, \\
\dot{x}_{2}=u_{2}, \\
\dot{x}_{3}=x_{1} u_{2}-x_{2} u_{1} .
\end{array}\right.
$$

This system is interesting for applications, since it models the motion of vehicles in suitable coordinates (see e.g. $[38,30]$ ). It is also a well known example of a controllable system for which the necessary Brockett's condition for the existence of a continuous stabilizing control 
law does not hold. The application of discontinuous stabilizing controller is then unavoidable. Our method provides a new discontinuous stabilizing control law, alternative to other control strategies (see e.g. [26, 32] and references therein). Suppose that the input constraint is $U(x)=U=\left\{u \in \mathbb{R}^{2}:\|u\|_{\infty} \leq 1\right\}$. The Brockett integrator satisfies Assumption 2.1 with

$$
g(x)=0, \quad h(x)=\left[\begin{array}{cc}
1 & 0 \\
0 & 1 \\
-x_{2} & x_{1}
\end{array}\right], \quad \begin{array}{ll}
M=\left[\begin{array}{cccc}
1 & 0 & -1 & 0 \\
0 & 1 & 0 & -1
\end{array}\right]^{T}, \\
N(x)=\left[\begin{array}{llll}
1 & 1 & 1 & 1
\end{array}\right]^{T} .
\end{array}
$$

7.1. Convex level set $\Omega$. Consider first the convex set $\Omega=\left\{x \in \mathbb{R}^{3}: H x \leq 1\right\}$, with

$$
H=\left[\begin{array}{cccccc}
1 & 1 & -1 & -1 & 0 & 0 \\
1 & -1 & 1 & -1 & 0 & 0 \\
0 & 0 & 0 & 0 & 1 & -1
\end{array}\right]^{T}
$$

which is a cube in $\mathbb{R}^{3}$ and consider the upper horizontal facet related to $H_{k}=H_{5}=\left[\begin{array}{lll}0 & 0 & 1\end{array}\right]$, that is $\mathscr{K}=\{5\}$, and the region $R_{\mathscr{K}}=\left\{x \in \mathbb{R}^{3}: x_{3}>\max \left\{\left|x_{1}\right|,\left|x_{2}\right|\right\}\right\}$. From Theorem 6.10, the nontrivial vertices of the dual problem feasibility region are

$$
\left\{\begin{array} { l } 
{ \delta H _ { 5 } h ( x ) + \sum _ { j = 1 } ^ { n _ { u } } \sigma _ { j } M _ { j } = 0 } \\
{ \delta = 1 , } \\
{ \sigma \geq 0 , }
\end{array} \Rightarrow \left\{\begin{array}{l}
-x_{2}+\sigma_{1}-\sigma_{3}=0 \\
x_{1}+\sigma_{2}-\sigma_{4}=0 \\
\sigma \geq 0
\end{array}\right.\right.
$$

with $\beta=0$. The extremes depend on $x_{1}$ and $x_{2}$. By symmetry, we restrict the analysis to $x \geq 0$. The extremes are given by $\sigma^{p}=\left[\begin{array}{llll}x_{2} & 0 & 0 & x_{1}\end{array}\right]^{T}$ and, from (6.14), the optimal control is

$$
\left\{\begin{array}{lll}
u_{1}^{*}=1, & u_{2}^{*}=-1, & \text { if } x_{1}>0, x_{2}>0 \\
u_{1}^{*} \in[-11], & u_{2}^{*}=-1, & \text { if } x_{1}>0, x_{2}=0 \\
u_{1}^{*}=1, & u_{2}^{*} \in[-11], & \text { if } x_{1}=0, x_{2}>0 \\
u_{1}^{*} \in[-11], & u_{2}^{*} \in[-11], & \text { if } x_{1}=0, x_{2}=0 .
\end{array}\right.
$$

This means that if $\left[\begin{array}{ll}x_{1} & x_{2}\end{array}\right]^{T}$ is in the interior of the first orthant, the maximal decreasing rate is given by $u=[1-1]^{T}$, if $x_{1}$ (resp. $x_{2}$ ) is zero then any admissible input such that $u_{2}^{*}=-1$ (resp. $u_{1}^{*}=1$ ) is optimal. Finally if $x_{1}=x_{2}=0$ every admissible input is optimal, indeed leading to an horizontal direction, hence tangent to the surface. Any selection of such a control law ensures that the decreasing rate $\lambda$ of $\Phi_{\Omega}(x)$ is attained for all $x \in R_{\mathscr{K}}$ such that:

$$
\begin{aligned}
& L_{\mathscr{K}}^{\lambda}\left(\beta^{p}, \delta^{p}, \sigma^{p} ; x\right)=\sum_{i=1}^{6} \beta_{i}^{p} H_{i} x+\delta^{p} H_{5} g(x)+\delta^{p}(\lambda+1) H_{5} x-\sum_{j=1}^{4} \sigma_{j}^{p} N_{j}(x) \leq H_{5} x, \\
& \Leftrightarrow \quad(\lambda+1) H_{5} x-\sum_{j=1}^{4} \sigma_{j}^{p} N_{j}(x) \leq H_{5} x, \quad \Leftrightarrow \quad \lambda x_{3} \leq x_{1}+x_{2},
\end{aligned}
$$

from Theorem 6.8 and since the other extremes lead to constraints $L_{\mathscr{K}}^{\lambda}\left(\beta^{q}, \delta^{q}, \sigma^{q} ; x\right) \leq H_{5} x$ satisfied in $R_{\mathscr{K}}$. The geometrical meaning of (7.2) is that there is no direction of the differential inclusion heading sufficiently downward for ensuring a decreasing rate $\lambda$ of the function $\Phi_{\Omega}(x)$ if $x \in R_{\mathscr{K}}$ is too close to axis $x_{3}$. The smaller is $\lambda$, the bigger is the region in which such a contraction can be assured, as expected, and the null rate, i.e. $\lambda=0$, is guaranteed in the whole $R_{\mathscr{K}}$. This is reasonable: in fact, $\dot{x}_{3}$ can be done negative by appropriately selecting the signs of $u_{1}$ and $u_{2}$ and its maximal modulus is $\left|x_{1}\right|+\left|x_{2}\right|$. That is, the maximal modulus of the vertical component of $\dot{x}$ is proportional to the distance (induced by the 1-norm) of $x$ 
from the $x_{3}$ axis. This implies also that the system moves horizontally if $x_{1}=x_{2}=0$ and then stability can be assured, by posing $\lambda=0$, but not the decreasing of the function $\Phi_{\Omega}$.

Consider $\mathscr{K}=\{1\}$, that is related to facet determined by the normal vector $H_{1}=\left[\begin{array}{ll}1 & 1\end{array}\right]$, and then such that $R_{\mathscr{K}}=\left\{x \in \mathbb{R}^{3}: x_{1}>0, x_{2}>0,\left|x_{3}\right|<x_{1}+x_{2}\right\}$. The nontrivial extreme

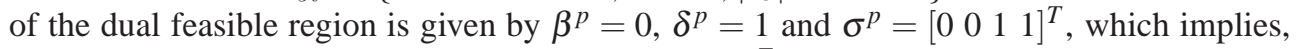
from (6.14), that the optimal control is $u^{*}=[-1-1]^{T}$. Moreover, by computing the region in which the contraction rate $\lambda$ is attainable for $\Phi_{\Omega}$ along one solution to the differential inclusion, i.e. where $L_{\mathscr{K}}^{\lambda}\left(\beta^{p}, \delta^{p}, \sigma^{p} ; x\right) \leq H_{1} x$, we obtain the constraint $\lambda\left(x_{1}+x_{2}\right) \leq 2$.

Finally consider the intersection between the upper horizontal facet and the vertical one in the first orthant, i.e. the point at which $H_{1} x=H_{5} x=\Phi_{\Omega}(x)$ and then $\mathscr{K}=\{1,5\}$ and $R_{\mathscr{K}}=\left\{x \in \mathbb{R}^{3}: x>0, x_{3}=x_{1}+x_{2}\right\}$. From Theorem 6.10, the nontrivial extremes are given by $\beta^{p}=0$ and the extremes of (6.8) which result in this case

$$
\left\{\begin{array} { l } 
{ \delta _ { 1 } H _ { 1 } h ( x ) + \delta _ { 5 } H _ { 5 } h ( x ) + \sum _ { j = 1 } ^ { n _ { u } } \sigma _ { j } M _ { j } = 0 } \\
{ \delta _ { 1 } + \delta _ { 5 } = 1 , } \\
{ \sigma \geq 0 }
\end{array} \quad \Rightarrow \quad \left\{\begin{array}{l}
\delta_{1}-\delta_{5} x_{2}+\sigma_{1}-\sigma_{3}=0 \\
\delta_{1}+\delta_{5} x_{1}+\sigma_{2}-\sigma_{4}=0 \\
\delta_{1}+\delta_{5}=1 \\
\sigma \geq 0
\end{array}\right.\right.
$$

that is the feasibility region of dual problem (6.13). To obtain the optimal input we consider the extremes of the region (7.3) and check if the KKT conditions (6.10) can be satisfied. Among the extremes of (7.3) there are $\left[\begin{array}{llllll}\delta_{1} & \delta_{5} & \sigma_{1} & \sigma_{2} & \sigma_{3} & \sigma_{4}\end{array}\right]^{T}=\left[\begin{array}{llllll}0 & 1 & x_{2} & 0 & 0 & x_{1}\end{array}\right]^{T}$ and $\left[\begin{array}{llllll}\delta_{1} & \delta_{5} & \sigma_{1} & \sigma_{2} & \sigma_{3} & \sigma_{4}\end{array}\right]^{T}=\left[\begin{array}{llllll}1 & 0 & 0 & 0 & 1 & x_{1}\end{array}\right]^{T}$, which lead to the optimal control in $R_{\{1\}}$ and $R_{\{5\}}$, analyzed above. Consider the first case, i.e. $\left[\begin{array}{ll}\delta_{1} & \delta_{5}\end{array}\right]^{T}=\left[\begin{array}{ll}0 & 1\end{array}\right]^{T}$ and $\sigma=\left[\begin{array}{llll}x_{2} & 0 & 0 & x_{1}\end{array}\right]^{T}$ which implies $u^{*}=[1-1]^{T}$. Then the second and fourth constraints in (6.10) result in

$$
\left\{\begin{array} { l } 
{ u _ { 1 } ^ { * } + u _ { 2 } ^ { * } \leq \mu ^ { * } , } \\
{ x _ { 1 } u _ { 2 } ^ { * } - x _ { 2 } u _ { 1 } ^ { * } = \mu ^ { * } , }
\end{array} \quad \Rightarrow \quad \left\{\begin{array}{l}
0 \leq \mu^{*} \\
-x_{1}-x_{2}=\mu^{*}
\end{array}\right.\right.
$$

which has no solution since $x_{1}>0$ and $x_{2}>0$. Analogously, for $\left[\begin{array}{ll}\delta_{1} & \delta_{5}\end{array}\right]^{T}=\left[\begin{array}{ll}1 & 0\end{array}\right]^{T}$ and $\sigma=\left[\begin{array}{llll}0 & 0 & 1 & 1\end{array}\right]^{T}$ we have $u^{*}=\left[\begin{array}{ll}-1 & -1\end{array}\right]^{T}$ and then from (6.10)

$$
\left\{\begin{array} { l } 
{ x _ { 1 } u _ { 2 } ^ { * } - x _ { 2 } u _ { 1 } ^ { * } \leq \mu ^ { * } , } \\
{ u _ { 1 } ^ { * } + u _ { 2 } ^ { * } = \mu ^ { * } }
\end{array} \quad \Rightarrow \quad \left\{\begin{array}{l}
x_{2} \leq x_{1}+\mu^{*} \\
\mu^{*}=-2
\end{array}\right.\right.
$$

which means that $u^{*}=[-1-1]^{T}$ is optimal for $x>0$ and $x_{2} \leq x_{1}-2$, and the decreasing rate $\lambda$ is ensured for $\lambda\left(x_{1}+x_{2}\right) \leq 2$. Another potential extreme point of (6.10) is given by $\left[\begin{array}{ll}\delta_{1} & \delta_{5}\end{array}\right]^{T}=\left[\begin{array}{ll}x_{2}\left(1+x_{2}\right)^{-1} & \left(1+x_{2}\right)^{-1}\end{array}\right]^{T}$, and $\sigma=\left[\begin{array}{lll}0 & 0 & 0\end{array}\right]^{T}$, extreme of (7.3), which constraints the optimal input to have $u_{2}^{*}=-1$. In this case the other constraints in (6.10) give

$$
\left\{\begin{array}{l}
x_{1} u_{2}^{*}-x_{2} u_{1}^{*}=\mu^{*} \\
u_{1}^{*}+u_{2}^{*}=\mu^{*}
\end{array} \quad \Rightarrow \quad u_{1}^{*}=\frac{1-x_{1}}{1+x_{2}} .\right.
$$

Then, the optimal control in $R_{\{1,5\}}=R_{\mathscr{K}}=\left\{x \in \mathbb{R}^{3}: x>0, x_{3}=x_{1}+x_{2}\right\}$ generates a sliding motion on the surface $R_{\mathscr{K}}$, in fact such that $\dot{x}_{3}=\dot{x}_{1}+\dot{x}_{2}$. Moreover, considering the bounds on $u$ (the first constraints in (6.10)), the state-dependent input is optimal in the $R_{\mathscr{K}}$ if $x_{2} \geq x_{1}-2$, which is (the closure of) the complement (with respect to the region in which $x>0$ ) of the region in which $u^{*}=[-1-1]^{T}$ is optimal, see (7.4). The rate $\lambda$ is ensured for $\lambda\left(x_{1}+x_{2}\right) \leq 1$.

Summarizing, if the active constraint is the upper horizontal facet, the optimal control in the first orthant of $\mathbb{R}^{n}$ is (7.1), which makes $x_{3}$ decrease. The function $\Phi_{\Omega}$ decreases with a rate greater than $\lambda$ if the state is distant enough (in terms of 1-norm) from the $x_{3}$ axis. For $x_{1}=x_{2}=0$, no decreasing is possible since all the inputs generate directions tangent to the 


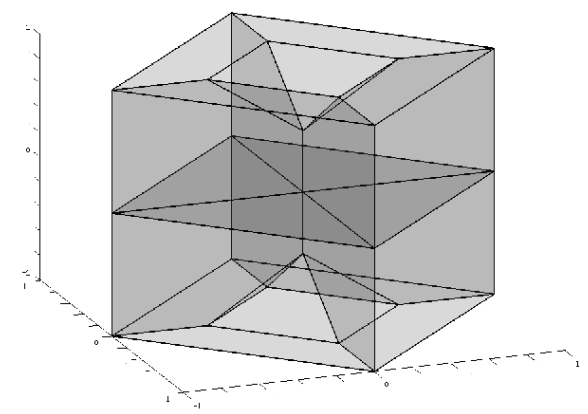

FIG. 3. Nonconvex level set $\Theta$.

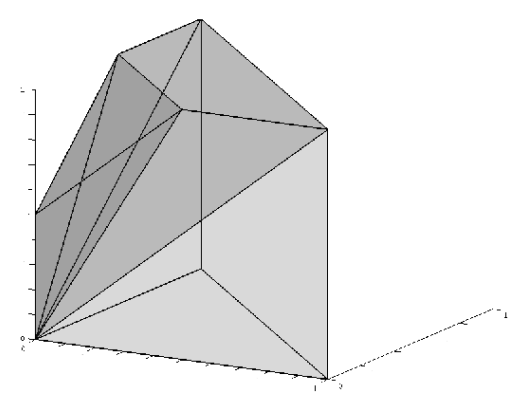

FIG. 4. Intersections of $\Theta$ with the first orthant.

level set of the function $\Phi_{\Omega}$. If the active constraint is $x_{1}+x_{2}=\Phi_{\Omega}(x)$ then the optimal control is $u^{*}=[-1-1]^{T}$ and the rate $\lambda$ is ensured if the state is not too far from the $x_{3}$ axis, i.e. if $\lambda\left(x_{1}+x_{2}\right) \leq 2$. Finally, if both constraints are active (i.e. $\left.x_{3}=x_{1}+x_{2}\right)$ then the optimal control is $u^{*}=\left[\frac{1-x_{1}}{1+x_{2}}-1\right]^{T}$, which makes the system slide on such a surface, if admissible. Otherwise, that is if $\frac{1-x_{1}}{1+x_{2}}<-1$, the optimal control is $u^{*}=[-1-1]^{T}$.

REMARK 7.1. For every $\lambda>0$, there is a region around the axis $x_{3}$ in which no admissible control generates one solution to the differential inclusion along which the function $\Phi_{\Omega}$ decreases and only stability can be guaranteed.

7.2. Nonconvex level set $\Theta$. To guarantee exponential stability, the convex level set $\Omega$ is replaced by $\Theta \in \mathscr{C}\left(\mathbb{R}^{n}\right)$ nonconvex. The resulting nonconvex set induces a control Lyapunov function which ensures exponential stability. Define the nonconvex function

$$
\Phi_{\Theta}(x)=\max \left\{\max _{i \in \mathbb{N}_{6}}\left\{H_{i}^{A} x\right\}, \min _{j \in \mathbb{N}_{4}}\left\{H_{j}^{B} x\right\}, \min _{k \in \mathbb{N}_{4}}\left\{-H_{k}^{B} x\right\}\right\}
$$

which is the function induced by the nonconvex set

$$
\Theta=\left(\bigcap_{i \in \mathbb{N}_{6}}\left\{x \in \mathbb{R}^{3}: H_{i}^{A} x \leq 1\right\}\right) \cap\left(\bigcup_{j \in \mathbb{N}_{4}}\left\{x \in \mathbb{R}^{3}: H_{j}^{B} x \leq 1\right\}\right) \cap\left(\bigcup_{k \in \mathbb{N}_{4}}\left\{x \in \mathbb{R}^{3}:-H_{k}^{B} x \leq 1\right\}\right) \text {. }
$$

with

$$
H^{A}=\left[\begin{array}{cccccc}
1 & 1 & -1 & -1 & 0 & 0 \\
1 & -1 & 1 & -1 & 0 & 0 \\
0 & 0 & 0 & 0 & 1 & -1
\end{array}\right]^{T}, \quad H^{B}=\left[\begin{array}{cccc}
-2 & 2 & -2 & 2 \\
-2 & -2 & 2 & 2 \\
2 & 2 & 2 & 2
\end{array}\right]^{T}
$$

To have a geometric perception of the set, define $A=\left\{x \in \mathbb{R}^{3}: H^{A} x \leq 1\right\}$, the unit box, and $B=\left\{x \in \mathbb{R}^{3}: \exists j \in \mathbb{N}_{4}\right.$, s.t. $\left.H_{j}^{B} x \leq 1\right\}$, which is the closure of the complement of the cone $\bar{B}=\left\{x \in \mathbb{R}^{3}: H^{B} x \geq 1\right\}$. Then $\Theta=A \cap B \cap(-B)$, which is represented in Figure 3. By symmetry, we restrict the analysis to the first orthant, see Figure 4, in which the constraint $H_{1}^{B} x=\left[\begin{array}{lll}-2 & -2 & 2\end{array}\right] x \leq 1$, is active for $x \geq 0$ such that $\left[\begin{array}{lll}1 & 1 & -0.5\end{array}\right] x \leq 0$. Consider such a constraint and such a region denoted with a slight abuse of notation $R_{\mathscr{K}}=R_{\left\{1^{B}\right\}}$ (the matrix $H^{\Theta}$ should be defined by concatenating $H^{A}, H^{B}$ and $-H^{B}$ and the indexes of $H^{\Theta}$ should be used to adequately define $\mathscr{K}$ ). The nontrivial extreme is given by for $\delta=1$ and $\beta=0$ and

$$
\left\{\begin{array}{r}
-2-2 x_{2}+\sigma_{1}-\sigma_{3}=0 \\
-2+2 x_{1}+\sigma_{2}-\sigma_{4}=0
\end{array}\right.
$$




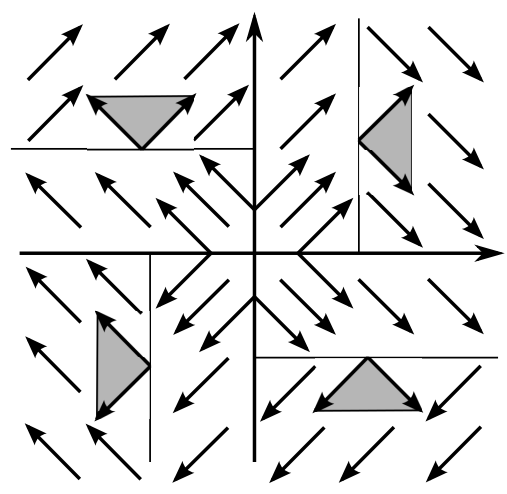

FIG. 5. Optimal control for $0.5\left|x_{3}\right|>\left|x_{1}\right|+$ $\left|x_{2}\right|$ with $x_{3} \geq 0$.

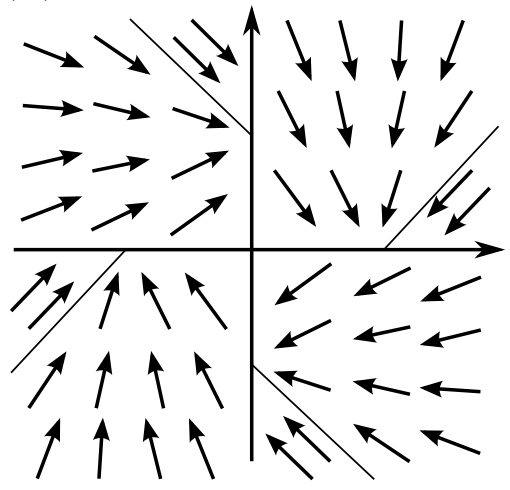

FIG. 7. Optimal control for $\left|x_{3}\right|=\left|x_{1}\right|+\left|x_{2}\right|$ with $x_{3} \geq 0$.

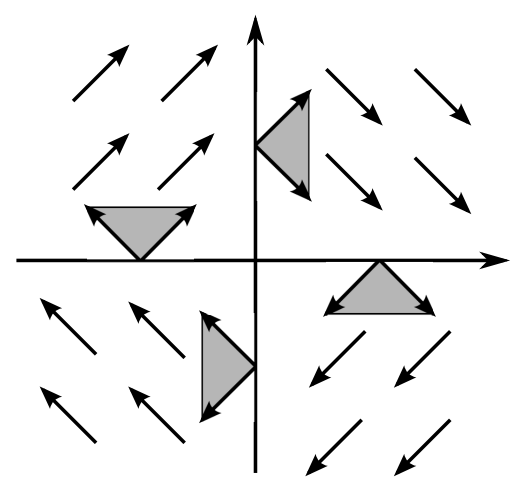

FIG. 6. Optimal control for $\left|x_{1}\right|+\left|x_{2}\right|<$ $\left|x_{3}\right|<2\left|x_{1}\right|+2\left|x_{2}\right|$ with $x_{3} \geq 0$.

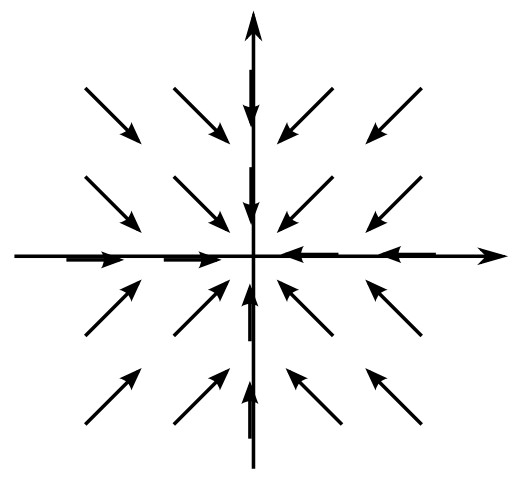

FIG. 8. Optimal control for $\left|x_{3}\right|<\left|x_{1}\right|+\left|x_{2}\right|$ with $x_{3} \geq 0$.

and three cases must be discriminated. The first concerns the region in which $x_{1}>1$ where the extreme is given by $\sigma=\left[\left(2+2 x_{2}\right) 00\left(-2+2 x_{1}\right)\right]^{T}$, the optimal control is $u^{*}=[1,-1]^{T}$ and it ensures the contraction rate $\lambda$ for all $x>0$ such that $\lambda\left(-x_{1}-x_{2}+x_{3}\right) \leq x_{1}+x_{2}$. In the region in which $x_{1}<1$, the extreme is $\sigma=\left[\left(2+2 x_{2}\right)\left(2-2 x_{1}\right) 00\right]^{T}$, the optimal control is $u^{*}=\left[\begin{array}{ll}1 & 1\end{array}\right]^{T}$ and it the contraction rate of $\lambda$ is attained at $\lambda\left(-x_{1}-x_{2}+x_{3}\right) \leq 2-x_{1}+x_{2}$. Finally, if $x_{1}=1$, then $\sigma=\left[\begin{array}{lllll}\left(2+2 x_{2}\right) & 0 & 0 & 0\end{array}\right]^{T}$ and every admissible $u^{*}$ with $u_{1}^{*}=1$ is optimal and guarantees decreasing rate $\lambda$ for $\lambda\left(-x_{1}-x_{2}+x_{3}\right) \leq 1+x_{2}$. We skip the analysis of the region in which the two constraints are both active, i.e. $H_{5}^{A} x=H_{1}^{B} x$, to concentrate our attention to the points at which the contingent cone is nonconvex.

Consider the $x$ such that $x_{1}>0, x_{2}=0$ and $x_{1}<0.5 x_{3}$. In this region the active constraints are the first two defining the set $B$ and then $\Theta_{I}(x)=\left\{x \in \mathbb{R}^{3}: H_{1}^{B} x \leq 1\right\} \cup\left\{x \in \mathbb{R}^{3}: H_{2}^{B} x \leq 1\right\}$ and the contingent epiderivative is $\Phi_{\Theta_{I}(x)}(y)=\min \left\{H_{1}^{B} y, H_{2}^{B} y\right\}$. Thus any $u \in U(x)$ for which either $H_{1}^{B}(g(x)+h(x) u+\lambda x) \leq 0$ or $H_{2}^{B}(g(x)+h(x) u+\lambda x) \leq 0$ is satisfied, is such that one solution to the differential inclusion exists along which the function $\Phi_{\Theta}(x)$ is decreasing with rate $\lambda$. This means that, both $u=\left[\begin{array}{ll}1 & 1\end{array}\right]^{T}$, optimal for $x>0$ (for $x_{1}<1$ ), and $u=\left[\begin{array}{ll}1-1\end{array}\right]^{T}$ optimal for $x_{1}>0, x_{2}<0$ and $x_{1}>3$, imply a decreasing rate $\lambda$. One should choose the $u^{*}$ that minimizes $\Phi_{\Theta_{I}(x)}(g(x)+h(x) u)$. Summarizing, the resulting optimal control in the different regions $R_{\left\{1^{B}\right\}}, R_{\left\{5^{A}\right\}}, R_{\left\{1^{A}, 5^{A}\right\}}$ and $R_{\left\{1^{A}\right\}}$ (and their analogous for all the orthants with $x_{3} \geq 0$ ) are depicted in while Figures 5, 6, 7 and 8, respectively.

REMARK 7.2. The Lyapunov conditions related to viability imply the existence of one 


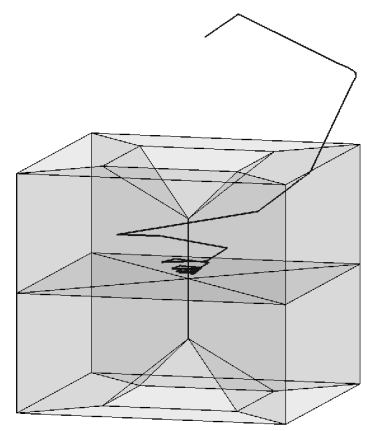

FIG. 9. Trajectory and set $\Theta$.

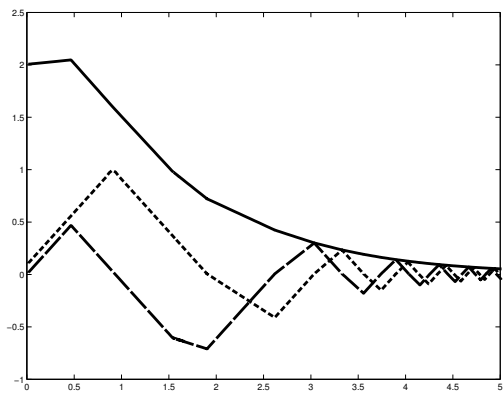

FIG. 11. State: $x_{1}$ (dotted), $x_{2}$ (dashed) and $x_{3}$ (solid).

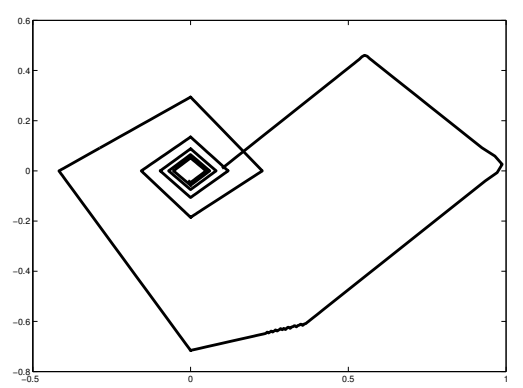

FIG. 10. Evolution of $x_{1}$ and $x_{2}$.

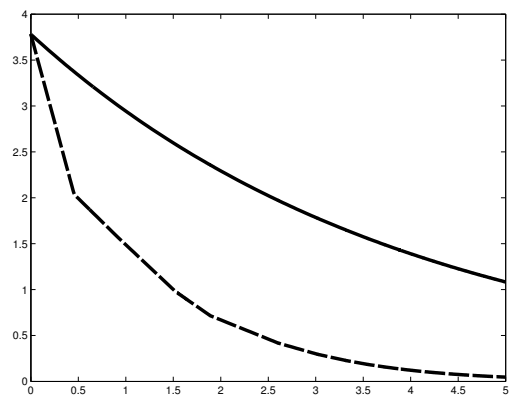

FIG. 12. Lyapunov (dashed) and exponential (solid) functions.

solution to the differential inclusion, that means the existence of a Caratheodory solution along which the induced function $\Phi_{\Theta}(x)$ decreases. The problem of determining whether all the trajectories (as discontinuous systems have not necessarily a unique solution, see [16]) are stable and converging to the origin, i.e. asymptotic stability in the sense of Filippov, is a possible extension of this work.

7.3. Simulation results. Finally, the simulation results are depicted in Figures 9-12. In Figure 9 the level set $\Theta$ and the trajectory of the controlled system with $x(0)=[0.1,0.01,2]^{T}$ are illustrated. The projection of the state on the plane $x_{3}=0$ is provided in Figure 10 and the evolution of the state in time in Figure 11. Notice that, although the value of $x_{3}$ increases at the beginning, the function $\Phi_{\Theta}(x)$ decreases, as shown in Figure 12 that reports also the exponential function with decreasing rate $\lambda=0.25$ for comparison.

8. Conclusions. This paper presented an approach to charaterize homogeneous control Lyapunov functions induced by certain nonconvex sets for nonlinear continuous-time systems with constrained input. A necessary and sufficient condition for local exponential stabilizability has been posed in form of convex constraints. Particular attention has been devoted to the numerical issues concerning the computation of the domain of attraction estimation and of the optimal control input. The example showed that nonconvexity might be an unavoidable feature to prove exponential stabilizability for nonlinear systems.

Other directions of research are possible: considering wider classes of nonconvex sets, leading to nonconvex optimization problems; tackling the problem of exponential stabilizability in the sense of Filippov; particularizing and applying the results to specific nonlinear systems; designing computational methods to generate the polytopes and the level sets for enlarging the domain of attraction. 


\section{REFERENCES}

[1] T. Alamo, A. Cepeda, M. Fiacchini, and E. F. Camacho, Convex invariant sets for discrete-time Lur'e systems, Automatica, 45 (2009), pp. 1066-1071.

[2] J.-P. AUBIN, A survey of viability theory, SIAM Journal of Control and Optimization, 28 (1990), pp. 749-788. [3] - Viability theory, Birkhäuser, 1991.

[4] J.-P. Aubin And H. FrankowsKa, Set-valued analysis, Birkhäuser, 1990.

[5] D. P. BERTSEKAS, Infinite-time reachability of state-space regions by using feedback control, IEEE Transactions on Automatic Control, 17 (1972), pp. 604-613.

[6] D. P. Bertsekas, A. Nedic, AND A. E. Ozdaglar, Convex analysis and optimization, Athena Scientific, 2003.

[7] F. BLANCHINI, Ultimate boundedness control for discrete-time uncertain systems via set-induced Lyapunov functions, IEEE Transactions on Automatic Control, 39 (1994), pp. 428-433.

[8] - Nonquadratic Lyapunov functions for robust control, Automatica, 31 (1995), pp. 451-461.

[9] F. Blanchini and S. Miani, Set-Theoretic Methods in Control, Birkhäuser, 2008.

[10] F. BlanCHINI AND C. SAVORgnan, Stabilizability of switched linear systems does not imply the existence of convex Lyapunov functions, Automatica, 44 (2008), pp. 1166 - 1170.

[11] S. Boyd and L. Vandenberghe, Convex Optimization, Cambridge University Press, 2004.

[12] R. W. BRockett, Asymptotic stability and feedback stabilization, in Differential geometric control theory, Birkhäuser, ed., 1983, pp. 181-191.

[13] E. B. Castelan And J. C. Hennet, On invariant polyhedra of continuous-time linear systems, IEEE Transactions on Automatic Control, 38 (1993), pp. 1680-1685.

[14] G. CHESI, Estimating the domain of attraction for non-polynomial systems via LMI optimizations, Automatica, 45 (2009), pp. 1536-1541.

[15] F. ClaRke, Discontinuous feedback and nonlinear systems, in Proc. of the 8th IFAC Symposium on Nonlinear Control Systems, 2010, pp. 1-29.

[16] J. CORTÉs, Discontinuous dynamical systems, IEEE Control Systems Magazine, 28 (2008), pp. 36-73.

[17] L. FARINA AND L. BENVENUTI, Invariant polytopes of linear systems, IMA Journal of Mathematical Control and Information, 15 (1998), pp. 233-240.

[18] M. Fiacchini, T. Alamo, And E.F. CAMACHo, On the computation of convex robust control invariant sets for nonlinear systems, Automatica, 46 (2010), pp. 1334-1338.

[19] — Invariant sets computation for convex difference inclusions systems, Systems and Control Letters, 61 (2012), pp. 819-826.

[20] M. FIACCHINI AND M. JUNGERS, Necessary and sufficient condition for stabilizability of discrete-time linear switched systems: A set-theory approach, Automatica, 50 (2014), pp. $75-83$.

[21] M. Fiacchini, S. TARbouriech, AND C. Prieur, Polytopic control invariant sets for differential inclusion systems: a viability theory approach, in Proc. of the American Control Conference, 2011, San Francisco, CA, USA, June 2011, pp. 1218-1223.

[22] — Quadratic stability for hybrid systems with nested saturations, IEEE Transactions on Automatic Control, 57 (2012), pp. 1832-1838.

[23] E. G. GILBERT AND K. TAN, Linear systems with state and control constraints: The theory and application of maximal output admissible sets, IEEE Transactions on Automatic Control, 36 (1991), pp. 1008-1020.

[24] R. GoebeL, Lyapunov functions and duality for convex processes, SIAM Journal on Control and Optimization, 51 (2013), pp. 3332-3350.

[25] J. M. GOMES DA SILVA JR. AND S. TARBOURIECH, Antiwindup design with guaranteed regions of stability: an LMI-based approach, IEEE Transactions on Automatic Control, 50 (2005), pp. 106-111.

[26] J. P. Hespanha, D. Liberzon, AND A. S. Morse, Logic-based switching control of a nonholonomic system with parametric modeling uncertainty, Systems \& Control Letters, 38 (1999), pp. 167-177.

[27] T. HU AND Z. Lin, Exact characterization of invariant ellipsoids for single input linear systems subject to actuator saturation, IEEE Transactions on Automatic Control, 47 (2002), pp. $164-169$.

[28] I. Kolmanovsky AND E. G. GilberT, Theory and computation of disturbance invariant sets for discretetime linear systems, Mathematical Problems in Engineering, 4 (1998), pp. 317-367.

[29] M. W. McConley, M. A. Dahleh, And E. Feron, Polytopic control Lyapunov functions for robust stabilization of a class of nonlinear systems, Systems \& Control Letters, 34 (1998), pp. 77-83.

[30] R. M. MURRAY AND S. S. SASTRY, Nonholonomic motion planning: steering using sinusoids, IEEE Transactions on Automatic Control, 38 (1993), pp. 700-716.

[31] S. Olaru, J. A. De Doná, M. M. Seron, and F. Stoican, Positive invariant sets for fault tolerant multisensor control schemes, International Journal of Control, 83 (2010), pp. 2622-2640.

[32] C. PRIEUR AND E. TRÉLAT, Robust optimal stabilization of the Brockett integrator via a hybrid feedback, Mathematical Control Signals Systems, 64 (2005), pp. 487-507.

[33] S. V. Raković, K. I. Kouramas, E. C. Kerrigan, J. C. Allwright, and D. Q. Mayne, The minimal robust positively invariant set for linear difference inclusions and its robust positively invariant 
approximations, tech. report, Imperial College London, 2005.

[34] A. RANTZER AND M. Johansson, Piecewise linear quadratic optimal control, IEEE Transactions on Automatic Control, 45 (2000), pp. 629-637.

[35] R. T. Rockafellar, Convex Analysis, Princeton University Press, USA, 1970.

[36] A. M. Rubinov AND A. A. YAGUBov, The space of star-shaped sets and its applications in nonsmooth optimization, in Quasidifferential Calculus, V.F. Demyanov and L.C.W. Dixon, eds., Springer Berlin Heidelberg, 1986, pp. 176-202.

[37] R. SchneIder, Convex bodies: The Brunn-Minkowski theory, vol. 44, Cambridge University Press, Cambridge, England, 1993.

[38] E. D. SonTAG, Stability and stabilization: discontinuities and the effect of disturbances, in Nonlinear Analysis, Differential Equations and Control, Kluwer, ed., 1999, pp. 551-598.

[39] S. TARbouriech, G. Garcia, J. M. Gomes DA Silva JR., AND I. QueInNEC, Stability and stabilization of linear systems with saturating actuators, Springer-Verlag, London, 2011. 